\title{
On the Sacks-Uhlenbeck flow of Riemannian surfaces
}

\author{
Min-Chun Hong and HaO Yin
}

\begin{abstract}
In this paper, we study an $\alpha$-flow for the Sacks-Uhlenbeck functional on Riemannian surfaces and prove that the limiting map of the $\alpha$-flow is a weak solution to the harmonic map flow. By an application of the $\alpha$-flow, we present a simple proof of an energy identity of a minimizing sequence in each homotopy class.
\end{abstract}

\section{Introduction}

Suppose that $M$ is a Riemannian manifold and $N$ is a closed manifold embedded in $\mathbb{R}^{k}$. A critical point $u$ of the Dirichlet energy

$$
E(u)=\int_{M}|\nabla u|^{2} d v
$$

is called a harmonic map.

Harmonic maps between Riemannian manifolds can be thought of as a natural generalization of geodesics, minimal surfaces and harmonic functions. A fundamental question on harmonic maps is: given a smooth map $u_{0}$ from $M$ to $N$, does there exists a smooth harmonic map representative in the homotopy class of $u_{0}$ ? In a pioneering work [7], Eells and Sampson answered this question in the case that the sectional curvature of $N$ is nonpositive by introducing the harmonic map flow to deform an initial map to a harmonic map in the same homotopy class. In general, one cannot expect to have the existence of smooth harmonic maps into general target manifolds due to the fact that singularities do occur. From now on, we assume that $M$ is a closed Riemannian surface; i.e., $\operatorname{dim} M=2$. Under certain topological conditions on $N$, the existence of minimizing harmonic maps in a homotopy class was proved by Lemaire [12] and Schoen and Yau [22]. In a well-known paper [19], Sacks and Uhlenbeck established many existence results of minimizing harmonic maps in their homotopy classes by introducing a family of 
functionals

$$
E_{\alpha}(u)=\int_{M}\left(1+|\nabla u|^{2}\right)^{\alpha} d v
$$

for $\alpha>1$. The $\alpha$-functional $E_{\alpha}$ is now called the 'Sacks-Uhlenbeck functional' and satisfies the Palais-Smale condition, so one can prove existence of critical points of $E_{\alpha}$ by either minimizing the energy functional or using Morse theory. Moreover, a bubbling phenomenon occurs by studying the limits of the critical points of $E_{\alpha}$ as $\alpha \rightarrow 1$ (see Section 4 in [19]). The method of the $\alpha$-functional has been very useful in studying many other problems. For example, Struwe in [23] used the $\alpha$-functional to show that for any closed surface $S \subset \mathbf{R}^{3}$, which is diffeomorphic to $S^{2}$, there is a non-trivial minimal surface intersecting $S$ orthogonally along its boundary.

On the other hand, Struwe [24] proved the global existence of the weak solution to the harmonic map flow and that the solution to the flow converges to a harmonic map as $t \rightarrow \infty$. Chang et al. [2] constructed an example where the harmonic map flow blows up at finite time, so in general the limiting harmonic map under the flow may not be in the same homotopy class as the initial map.

In this paper, we study an $\alpha$-flow for the perturbed energy $E_{\alpha}$ in the same homotopy class of the initial map. More precisely, we consider the following evolution problem:

$$
\partial_{t} u=\triangle_{M} u+(\alpha-1) \frac{\nabla|\nabla u|^{2} \cdot \nabla u}{1+|\nabla u|^{2}}+A(u)(\nabla u, \nabla u),
$$

with $u(x, 0)=u_{0}$, where $\triangle_{M}$ is the Laplacian operator with respect to the Riemannian metric of $M$ and $A$ is the second fundamental form of $N$. We call (1.1) the Sacks-Uhlenbeck flow (or $\alpha$-flow). We would like to point out that the $\alpha$-flow is not the standard gradient flow, but the flow has some analytic advantages.

Firstly, we have

Theorem 1.1. Let $u_{0}: M \rightarrow N$ be a smooth map. For sufficiently small $\alpha-1$, there exists a unique global smooth solution $u_{\alpha}(x, t)$ to the evolution problem (1.1) in $M \times[0, \infty)$ with initial value $u_{0}$. Moreover, for any $t_{i} \rightarrow \infty$, $u_{\alpha}\left(\cdot, t_{i}\right)$ converges smoothly to a limit map $u_{\alpha}$, which is a critical point of the Sacks-Uhlenbeck functional.

For each $\alpha>1$, let $u_{\alpha}(x, t)$ be the global smooth solution to (1.1) in the same homotopy class with the initial map $u_{0}$ from $M$ to $N$. It is a nature 
problem to study the limiting behaviour of the solution $u_{\alpha}(x, t)$ as $\alpha \rightarrow 1$ as in [19]. Thus, we prove

Theorem 1.2. (i) As $\alpha \rightarrow 1$, the solution $u_{\alpha}$ converges smoothly to $u$ on $M \times[0, \infty) \backslash \Sigma$, where the concentration set $\Sigma$ is a closed set defined by

$$
\Sigma=\bigcap_{0<R<R_{M}}\left\{z \in M \times[0, \infty) \mid \liminf _{\alpha \rightarrow 1} \Psi_{R}^{\alpha}\left(u_{\alpha}, z\right) \geq \varepsilon_{0}\right\}
$$

for some $\varepsilon_{0}$ to be determined. For the precise definition of $\Psi_{R}^{\alpha}$, see Section 3.

(ii) For any two positive $t_{1}$ and $t_{2}, \mathcal{P}^{2}\left(\Sigma \cap\left(M \times\left[t_{1}, t_{2}\right]\right)\right)$ is finite, where $\mathcal{P}^{2}$ denotes the 2-dimensional parabolic Hausdorff measure. Moreover, for any $t \in(0,+\infty), \quad \Sigma_{t}=\Sigma \cap(M \times\{t\})$ consists of at most finitely many points.

(iii) $u$ is a weak solution to the harmonic map flow.

In fact, this result is similar to the one using the Ginzburg-Landau flow approximation. In [4], Chen and Struwe used the Ginzburg-Landau flow approximation to construct a global weak solution of the harmonic map flow for any dimension larger than two. Further results obtained by the GinzburgLandau flow were discussed by Lin and Wang in [16]. The method of the Ginzburg-Landau flow is very powerful for showing the global existence and partial regularity of a weak solution to the harmonic map flow, but it seems that the flow approximation loses control on the topological quantity of maps. The advantage of the Sacks-Uhlenbeck flow approximation is that the solution $u_{\alpha}$ remains in the same homotopy class of $u_{0}$, therefore it seems that the Sacks-Uhlenbeck flow provides a nice geometric picture and can be used to produce some geometric applications (see Theorem 1.4 below).

With Theorem 1.2 in hand, it is very interesting to ask whether the limiting solution $u$ of the Sacks-Uhlenbeck flow is the global weak solution $\hat{u}$ constructed by Struwe in [24]. Although we do not have a complete answer to the question, we can compare some properties of $u$ with the solution $\hat{u}$ in the following:

Theorem 1.3. (i) The first concentration time for $u_{\alpha}$ as $\alpha \rightarrow 1$, i.e.,

$$
T=\inf _{(x, t) \in \Sigma} t
$$

is the same as the first singular time of the weak solution $\hat{u}$ constructed by Struwe. Hence, $u_{\alpha}$ converges smoothly to the solution $\hat{u}$ in $M \times[0, T)$. 
(ii) Consider the following two limits of measures:

$$
|\nabla \hat{u}(\cdot, t)|^{2} d v \rightarrow|\nabla \hat{u}(\cdot, T)|^{2} d v+\sum_{i=1}^{\hat{k}} \hat{m}_{i} \delta_{\hat{p}_{i}} \quad \text { as } t \rightarrow T,
$$

and

$$
\left(1+\left|\nabla u_{\alpha}(\cdot, T)\right|^{2}\right)^{\alpha} d v \rightarrow\left(1+|\nabla u(\cdot, T)|^{2}\right) d v+\sum_{i=1}^{k} m_{i} \delta_{p_{i}} \quad \text { as } \alpha \rightarrow 1 .
$$

Then $k=\hat{k}, p_{i}=\hat{p}_{i}$ and $\hat{m}_{i} \geq m_{i}$.

Without the assumption of the energy inequality, weak solutions to the heat flow for harmonic maps may not always be unique (see [1] and [26]), so we cannot exclude the possibility that the weak solution $u$ is different from the solution $\hat{u}$ constructed in [24] after the first singular time $T$. On this issue, we would like to make the following remark: Consider maps from $S^{2}$ to itself. According to [2], there exists an initial value map $u_{0}$ of degree three such that Struwe's solution of the harmonic map flow blows up at some finite time $T$ and then the homotopy class of the solution is changed for $t>T$. Hence, the solutions $u_{\alpha}$ of the $\alpha$-flow cannot converge strongly to Struwe's solution after $T$. A natural question is what we can say about $\Sigma$ (See [13]).

In the final part of this paper, we apply the $\alpha$-flow to study a minimizing sequence in a given homotopy class. Let $u_{i}$ be a sequence of smooth maps minimizing $E(u)=\int_{M}|\nabla u|^{2} d v$ in a fixed homotopy class of maps. Since $u_{i}$ is bounded in $W^{1,2}$, there is a weak limit $u$ in $W^{1,2}(M, N)$. In general, $u$ may not be in the same homotopy class, but we can show:

Theorem 1.4. Let $u$ be the weak limit of the above minimizing sequence $\left\{u_{i}\right\}$. Then it is a harmonic map from $M$ to $N$ and there exist harmonic maps $\omega_{k}: S^{2} \rightarrow N$ with $k=1, \ldots, l$ such that

$$
\lim _{i \rightarrow \infty} E\left(u_{i}\right)=E(u)+\sum_{k=1}^{l} E\left(\omega_{k}\right) .
$$

Moreover, if $\pi_{2}(N)$ is trivial, then $u_{i}$ converges strongly to $u$ in $W^{1,2}(M, N)$ and $u$ is a minimizer in the homotopy class of $u_{i}$.

The last part of this theorem can be compared with Theorem 5.1 of [19], where under the same assumption that $\pi_{2}(N)$ is trivial, the existence of a 
minimizer in each homotopy class is proved (see also $[12,22]$ ). We improve the result a little by obtaining that every smooth minimizing sequence converges to such a minimizer. In the proof, we use the $\alpha$-flow to modify the original minimizing sequence and study the blow-up of the new sequence as in Theorem 5.1 of [19]. The energy identity of minimizers of the SacksUhlenbeck functional was implicitly established by Chen and Tian in [3]. Our proof of the energy identity is different from the one in [3]. Although the result of Theorem 1.4 may be regarded as a consequence of the theory developed by Duzaar and Kuwert [6], the advantage is that we can avoid the use of the concept of "weak homotopy class" in the Sobolev space $W^{1,2}(M, N)$, which is formulated using big machinery. Furthermore, we would like to mention that the related energy identity of critical points of the Sacks-Uhlenbeck functionals was recently discussed by Li and Wang [14], and by Lamm [11].

Remark 1.5. After we finished the first version of this paper, Yuxiang Li informed us that in [15] they used a similar idea to show the energy identify for a sequence of minimizers of $E_{\alpha}$ as $\alpha \rightarrow 1$. In fact, while Li and Wang in [15] used a reduction procedure of Ding and Tian [5], we use the bubble tree construction of Parker in [18] to find the connecting geodesics, so our approach is different. Our result is also stronger than theirs.

Finally, we would like to mention some results about the approximation method of harmonic maps in a homotopy class for higher dimensional manifolds (e.g. see $[9,28]$ ).

The paper is organized as follows. In Section 2, we prove Theorem 1.1. In Section 3, we give a proof of Theorem 1.2. In Section 4, we complete a proof of Theorem 1.3 except for the energy identity, which is proved in Section 5.

\section{Global existence and convergence of $\alpha$-flow}

This section is devoted to the proof of Theorem 1.1. The system of the $\alpha$-flow (1.1) is a non-linear parabolic system. For a smooth initial value $u_{0}$, there is a local smooth solution to the system (see below appendix for details in Appendix A); i.e., there exists $T>0$ and a smooth solution $u(x, t)$ defined on $[0, T)$. In fact, the $\alpha$-flow (1.1) can be written as follows:

$$
\begin{aligned}
\left(1+|\nabla u|^{2}\right)^{\alpha-1} \partial_{t} u= & \operatorname{div}\left(\left(1+|\nabla u|^{2}\right)^{\alpha-1} \nabla u\right) \\
& +\left(1+|\nabla u|^{2}\right)^{\alpha-1} A(u)(\nabla u, \nabla u) .
\end{aligned}
$$

The first global estimate is: 
Lemma 2.1. Let $u(x, t)$ be a solution to the $\alpha$-flow in $M \times[0, T)$. Then we have

$$
E_{\alpha}(u(\cdot, 0))=E_{\alpha}(u(\cdot, t))+\int_{0}^{t} \int_{M}\left(1+|\nabla u|^{2}\right)^{\alpha-1}\left|\partial_{t} u\right|^{2} d v d t
$$

for any $t \in[0, T)$.

Proof. The result follows from multiplying (2.1) by $\partial_{t} u$ and integrating by parts.

The second estimate is a local version of the energy inequality:

Lemma 2.2. Let $u$ be a solution to the $\alpha$-flow. For any constant $R>0$ and any point $x \in M$, we have

$$
\int_{B_{R}(x)}\left(1+\left|\nabla u\left(\cdot, t_{2}\right)\right|^{2}\right)^{\alpha} d v \leq \int_{B_{2 R}(x)}\left(1+\left|\nabla u\left(\cdot, t_{1}\right)\right|^{2}\right)^{\alpha} d v+C \frac{t_{2}-t_{1}}{R^{2}} E_{0}
$$

for all $t_{1}, t_{2} \in[0, T)$ with $t_{1} \leq t_{2}$, where $E_{0}$ is an upper-bound of the overall energy.

Proof. Let $\varphi$ be a cut-off function supported in $B_{R}(x)$ with $\varphi \equiv 1$ on $B_{R / 2}(x)$. Multiplying Equation (2.1) by $\partial_{t} u \varphi^{2}$, we obtain

$$
\int_{B_{R}(x)}\left(1+\left|\nabla u\left(\cdot, t_{2}\right)\right|^{2}\right)^{\alpha} d v \leq \int_{B_{2 R}(x)}\left(1+\left|\nabla u\left(\cdot, t_{1}\right)\right|^{2}\right)^{\alpha} d v+C \frac{t_{2}-t_{1}}{R^{2}} E_{0} .
$$

One of key steps in proving Theorem 1.1 is to derive a Bochner-type formula. Let $\left(g_{i j}\right)$ be a Riemannian metric on $M$. Then

Lemma 2.3. (Bochner's type formula) Let $u(x, t)$ be a smooth solution to the $\alpha$-flow (1.1) and set $e(u):=|\nabla u|^{2}$. Then, for $\alpha-1$ sufficiently small, we have

$$
\begin{aligned}
& \partial_{t} e(u)-\frac{1}{\sqrt{|g|}} \frac{\partial}{\partial x_{i}}\left(\sqrt{|g|}\left(g^{i j}+\frac{(\alpha-1)}{1+|\nabla u|^{2}} g^{i k} \frac{\partial u^{\beta}}{\partial x_{k}} g^{j l} \frac{\partial u^{\beta}}{\partial x_{l}}\right) \frac{\partial e(u)}{\partial x_{j}}\right) \\
& \quad \leq C e(u)(e(u)+1)
\end{aligned}
$$

where the constant $C$ does not depend on $\alpha$ and $u$. 
Proof. In a neighbourhood of each point $x \in M$, we can choose an orthonormal frame $\left\{e_{1}, e_{2}\right\}$. We denote by $\nabla_{i}$ the first covariant derivative with respect to $e_{i}$ and by $u_{j i}$ the second covariant derivatives of $u$ and so on. In a local frame, we have

$$
\nabla_{j} e(u)=2 u_{k}^{\gamma} u_{k j}^{\gamma}, \quad\left|\nabla^{2} u\right|^{2}=\sum_{k, i, \gamma}\left|u_{k i}^{\gamma}\right|^{2}
$$

Then we have

$$
\begin{aligned}
& \nabla_{i}\left(\left(\delta_{i j}+2(\alpha-1) \frac{u_{i}^{\beta} u_{j}^{\beta}}{1+|\nabla u|^{2}}\right) \nabla_{j} \mathrm{e}(u)\right) \\
& \quad=2 \nabla_{i}\left(u_{k}^{\gamma} u_{k i}^{\gamma}+2(\alpha-1) \frac{u_{i}^{\beta} u_{j}^{\beta} u_{k}^{\gamma} u_{k j}^{\gamma}}{1+|\nabla u|^{2}}\right) \\
& \quad \geq 2\left|\nabla^{2} u\right|^{2}+2 u_{k}^{\gamma} u_{i i k}^{\gamma}+4(\alpha-1) \nabla_{i}\left(\frac{u_{i}^{\gamma} u_{k}^{\gamma} u_{j}^{\beta} u_{k j}^{\beta}}{1+|\nabla u|^{2}}\right)-C e(u) \\
& \quad \geq \frac{3}{2}\left|\nabla^{2} u\right|^{2}+2 u_{k}^{\gamma} u_{i i k}^{\gamma}+4(\alpha-1) \frac{u_{i}^{\gamma} u_{k}^{\gamma} u_{j}^{\beta} u_{k j i}^{\beta}}{1+|\nabla u|^{2}}-C e(u) \\
& \quad \geq\left|\nabla^{2} u\right|^{2}+2 u_{k}^{\gamma} \nabla_{k}\left(u_{i i}^{\gamma}+2(\alpha-1) \frac{u_{i}^{\gamma} u_{j}^{\beta} u_{j i}^{\beta}}{1+|\nabla u|^{2}}\right)-C e(u) .
\end{aligned}
$$

Here we have twice used the Ricci identity for switching third-order derivatives. Using (1.1), we have

$$
\begin{aligned}
& \frac{\partial}{\partial t} e(u)-\nabla_{i}\left(\left(\delta_{i j}+2(\alpha-1) \frac{u_{i}^{\beta} u_{j}^{\beta}}{1+|\nabla u|^{2}}\right) \nabla_{j} e(u)\right) \\
& \quad \leq-\left|\nabla^{2} u\right|^{2}-2 u_{k}^{\gamma} \nabla_{k}\left(A^{\gamma}(u)(\nabla u, \nabla u)\right)+C e(u) \\
& \quad \leq C e(u)(e(u)+1) .
\end{aligned}
$$

This proves our claim.

Although the Bochner formula is motivated by the result of Uhlenbeck [27] on $p$-harmonic functions, it seems that this type of Bochner's formula is new. Using the formula, we can show a small $\epsilon$-regularity estimate by following a method of Schoen [21] and Struwe [25]. More precisely, we have 
Lemma 2.4. Let $u(x, t)$ be a solution of the $\alpha$-flow in $M \times[0, T)$ for a $T>0$. There is a small constant $\varepsilon_{0}>0$ such that if

$$
\sup _{0 \leq t<T} \int_{B_{r_{0}}\left(x_{0}\right)}|\nabla u(x, t)|^{2} d x \leq \varepsilon_{0}
$$

for a point $\left(x_{0}, t_{0}\right) \in M \times(0, T)$ with $r_{0}^{2} \leq T$, then

$$
|\nabla u(x, t)| \leq \frac{C}{r_{0}^{2}}, \quad \forall(x, t) \in B_{r_{0} / 2}\left(x_{0}\right) \times\left[t_{0}-\frac{r_{0}^{2}}{4}, t_{0}\right],
$$

where the constant $C$ depends not on $(x, t)$.

Proof. Set

$$
P_{r}=\left\{(x, t) \mid x \in B_{r}\left(x_{0}\right) \quad \text { and } t_{0}-r^{2} \leq t \leq t_{0}\right\}
$$

We can find $\rho \in\left[0, r_{0}\right]$ such that

$$
\left(r_{0}-\rho\right)^{2} \sup _{P_{\rho}} e(u)=\max _{\sigma \in\left[0, r_{0}\right]}\left\{\left(r_{0}-\sigma\right)^{2} \sup _{P_{\sigma}} e(u)\right\} .
$$

Let $\left(x_{1}, t_{1}\right)$ be the point in $P_{\rho}$ such that

$$
e \triangleq e(u)\left(x_{1}, t_{1}\right)=\sup _{P_{\rho}} e(u)
$$

If $e\left(r_{0}-\rho\right)^{2} \leq 4$, then

$$
\left(\frac{r_{0}}{2}\right)^{2} \sup _{P_{r_{0} / 2}} e(u) \leq e\left(r_{0}-\rho\right)^{2} \leq 4,
$$

which means the lemma is proved. Hence, we may assume that $e\left(r_{0}-\rho\right)^{2}>4$. Set

$$
v(x, t)=u\left(x_{1}+\frac{x}{e^{1 / 2}}, t_{1}+\frac{t}{e}\right)
$$

for $(x, t) \in B_{1}(0) \times[-1,0]$, so $v$ satisfies the scaled $\alpha$-flow equation

$$
\partial_{t} v=\frac{\operatorname{div}\left(\left(e^{-1}+|\nabla v|^{2}\right)^{\alpha_{k}-1} \nabla v\right)}{\left(e^{-1}+|\nabla v|^{2}\right)^{\alpha_{k}-1}}+A(v)(\nabla v, \nabla v) .
$$


By the scaling invariance of the Dirichlet energy, we have

$$
\sup _{t \in[-1,0]} \int_{B_{1}(0)} e(v) d v \leq \varepsilon_{0}
$$

Moreover, we have $e(v)(0,0)=1$ and

$$
\sup _{B_{1}(0) \times[-1,0]} e(v) \leq e^{-1} \sup _{\frac{P_{\frac{r_{0}+\rho}{2}}}{2}} e(u) \leq e^{-1} \frac{e\left(r_{0}-\rho\right)^{2}}{\left(r_{0}-\frac{r_{0}+\rho}{2}\right)^{2}} \leq 4 .
$$

By Lemma 2.3 and (2.5), we have

$$
\frac{\partial}{\partial t} e(v)-\nabla_{i}\left(a_{i j}(v) \nabla_{j} e(v)\right) \leq C e(v)
$$

where

$$
a_{i j}(v)=\delta_{i j}+2(\alpha-1) \frac{v_{i}^{\beta} v_{j}^{\beta}}{e^{-1}+|\nabla v|^{2}} .
$$

The symmetric matrix $\left(a_{i j}(v)\right)$ has eigenvalues satisfying the uniform elliptic condition. By a well-known Moser iteration and (2.4), we have

$$
1=e(v)(0,0) \leq C\left(\int_{B_{1}(0) \times[-1,0]} e(v)^{2}\right)^{1 / 2} .
$$

By (2.5), we obtain

$$
1 \leq C \int_{B_{1}(0) \times[-1,0]} e(v) \leq C \varepsilon_{0},
$$

which is impossible if we choose $\varepsilon_{0}$ small. This proves our claim.

For each fixed $\alpha>1$, the small energy assumption in Lemma 2.4 is automatically true because of the Hölder inequality and the uniform boundness of the energy $E_{\alpha}(u)$. More precisely, For any $t \in[0, T)$, Lemma 2.1 implies that

$$
\int_{M}\left(1+|\nabla u|^{2}\right)^{\alpha}(t) d v \leq C
$$

For each $\varepsilon_{0}>0$, there exists a constant $r_{0}>0$, depending on $\varepsilon_{0}$ and $\alpha$, such that for all $x \in M$ and $t<T$, we have

$$
\int_{B_{r_{0}}(x)}|\nabla u(\cdot, t)|^{2} d v \leq \varepsilon_{0} .
$$

We now complete the proof of Theorem 1.1. 
Proof. By the local existence, there is the unique smooth solution $u(x, t)$ of the alpha-flow in $M \times[0, T)$ for some $T>0$. we will show that the solution $u$ can be extended smoothly to the time $T$. By Lemma 2.4, we have the $C^{0}$-estimate for $\nabla u(x, t)$ for all $t \in[0, T)$. Next, we show higher-order derivative estimates. For $x_{0} \in M$ and $T / 2<t_{0}<T$, set $P_{r}=B_{r}\left(x_{0}\right) \times\left[t_{0}-r^{2}, t_{0}\right]$. Suppose $\varphi$ is a cut-off function supported in $P_{T / 2}$ with $\varphi \equiv 1$ in $P_{T / 4}$. Multiplying (1.1) by $\varphi$, we obtain

$$
\partial_{t}(u \varphi)-\triangle(u \varphi)=(\alpha-1) \frac{\left(\nabla^{2}(u \varphi), \nabla u\right) \nabla u}{1+|\nabla u|^{2}}+\mathcal{R}[\varphi, u, \nabla u],
$$

where $\mathcal{R}$ is a term involving $u, \nabla u$ and derivatives of $\varphi$. By Lemma 2.4 and the $L^{p}$ estimate of linear parabolic equations, we have

$$
\|\varphi u\|_{W^{2, p}\left(P_{T / 2}\right)} \leq C(\alpha-1)\left\|\nabla^{2}(\varphi u)\right\|_{L^{p}\left(P_{T / 2}\right)}+C,
$$

for any $p>0$. When $\alpha-1$ is small,

$$
\|\varphi u\|_{W^{2, p}\left(P_{T / 2}\right)} \leq C .
$$

We then take one more space derivative of (2.6) to get

$$
\partial_{t}(\varphi \nabla u)-\triangle(\varphi \nabla u)=(\alpha-1) \frac{\left(\nabla^{3}(\varphi u), \nabla u\right) \nabla u}{1+|\nabla u|^{2}}+\mathcal{Q}\left[\varphi, u, \nabla u, \nabla^{2} u\right] .
$$

Here $\mathcal{Q}$ involves $u, \nabla u, \nabla^{2} u$ and derivatives of $\varphi$. Using the $L^{p}$ estimate again, we have (when $\alpha-1$ is sufficiently small)

$$
\|\varphi u\|_{W^{3, p}\left(P_{T / 2}\right)} \leq C
$$

for any $p$. Therefore,

$$
\left\|\partial_{t} \nabla(\varphi u)\right\|_{L^{p}\left(P_{T / 2}\right)}, \quad\left\|\nabla^{2}(\varphi u)\right\|_{L^{p}\left(P_{T / 2}\right)} \leq C .
$$

For $p>3$, the Sobolev embedding theorem yields that

$$
\|\nabla(\varphi u)\|_{C^{\beta}\left(P_{T / 2}\right)} \leq C
$$

for some $\beta>0$. We can now apply the Schauder theory for parabolic equations to obtain higher-order estimates, which means that we can extend the solution $u(x, t)$ smoothly to $t=T$. Then the local existence result for smooth initial data implies $T=\infty$. 


\section{The limiting behaviour of the Sacks-Uhlenbeck flow}

For a fixed initial map $u_{0}$, it follows from Theorem 1.1 that there is a unique global solution $u_{\alpha}$ to $(1.1)$ in $M \times[0, \infty)$ for each $\alpha>1$. In this section, we study the limit of the solutions $u_{\alpha}$ as $\alpha \rightarrow 1$. We start with two lemmas. The first one is another Bochner-type formula.

Lemma 3.1. Let $u(x, t)$ be a classical solution to the $\alpha$-flow (1.1) and set $e_{\alpha}(u):=\left(1+|\nabla u|^{2}\right)^{\alpha}$. If $\alpha-1$ is sufficiently small, then there is a constant $C>0$ such that

$$
\frac{\partial}{\partial t} e_{\alpha}(u)-\nabla_{i}\left(\left(\delta_{i j}+2(\alpha-1) \frac{u_{i}^{\beta} u_{j}^{\beta}}{1+|\nabla u|^{2}}\right) \nabla_{j} e_{\alpha}(u)\right) \leq C e_{\alpha}(u)\left(1+|\nabla u|^{2}\right)
$$

Proof. By direct computation and switching indices as in Lemma 2.3, we have

$$
\begin{aligned}
\nabla_{i} & \left(\left(\delta_{i j}+2(\alpha-1) \frac{u_{i}^{\beta} u_{j}^{\beta}}{1+|\nabla u|^{2}}\right) \nabla_{j} e_{\alpha}(u)\right) \\
= & 2 \alpha \nabla_{i}\left(\left(1+|\nabla u|^{2}\right)^{\alpha-1} u_{k}^{\gamma} u_{k i}^{\gamma}+2(\alpha-1)\left(1+|\nabla u|^{2}\right)^{\alpha-2} u_{i}^{\gamma} u_{k}^{\gamma} u_{j}^{\beta} u_{k j}^{\beta}\right) \\
= & 2 \alpha \nabla_{i}\left[u_{k}^{\gamma} \nabla_{k}\left(u_{i}^{\gamma}\left(1+|\nabla u|^{2}\right)^{\alpha-1}\right)\right] \\
= & 2 \alpha\left|u_{k i}^{\gamma}\right|^{2}\left(1+|\nabla u|^{2}\right)^{\alpha-1}+4 \alpha(\alpha-1)\left(1+|\nabla u|^{2}\right)^{\alpha-2} u_{k i}^{\gamma} u_{j k}^{\beta} u_{i}^{\gamma} u_{j}^{\beta} \\
& +2 \alpha u_{k}^{\gamma} \nabla_{i} \nabla_{k}\left(u_{i}^{\gamma}\left(1+|\nabla u|^{2}\right)^{\alpha-1}\right) .
\end{aligned}
$$

Again, we assume $\alpha-1$ is small whenever necessary. Using the Ricci identity to switch the order of covariant derivatives, we have

$$
\begin{aligned}
\nabla_{i} & \left(\left(\delta_{i j}+2(\alpha-1) \frac{u_{i}^{\beta} u_{j}^{\beta}}{1+|\nabla u|^{2}}\right) \nabla_{j} e_{\alpha}(u)\right) \\
\geq & 2 \alpha\left|u_{k i}^{\gamma}\right|^{2}\left(1+|\nabla u|^{2}\right)^{\alpha-1}+2 \alpha u_{k}^{\gamma} \nabla_{k} \nabla_{i}\left(u_{i}^{\gamma}\left(1+|\nabla u|^{2}\right)^{\alpha-1}\right) \\
& -C\left(1+|\nabla u|^{2}\right)^{\alpha} \\
\geq & \alpha\left|u_{k i}^{\gamma}\right|^{2}\left(1+|\nabla u|^{2}\right)^{\alpha-1}+2 \alpha\left(1+|\nabla u|^{2}\right)^{\alpha-1} u_{k}^{\gamma} \nabla_{k} \frac{\partial u}{\partial t} \\
& +2 \alpha\left(1+|\nabla u|^{2}\right)^{\alpha-1} u_{k}^{\gamma} \nabla_{k}\left(A^{\gamma}(u)(\nabla u, \nabla u)\right)-C\left(1+|\nabla u|^{2}\right)^{\alpha} \\
\geq & \frac{1}{2}\left|u_{k i}^{\gamma}\right|^{2}\left(1+|\nabla u|^{2}\right)^{\alpha-1}+\frac{\partial}{\partial t} e_{\alpha}(u)-C e_{\alpha}(u)\left(1+|\nabla u|^{2}\right) .
\end{aligned}
$$


Here in the last two inequalities, we have used the equation of $u$ and the fact that $\alpha-1$ is small.

The second lemma is a parabolic monotonicity formula. Such a formula for the harmonic map flow was first established by Struwe in [25] for the Euclidean case and by Chen-Struwe in [4] for general case. Let $u$ be a solution to (1.1). Suppose $i_{M}$ is the injectivity radius of $M$. For a fixed point $x_{0}$ in $M$, choose the normal coordinates $\left\{x_{i}\right\}$ and a cut-off function $\varphi$ supported in $B_{i_{M}}\left(x_{0}\right)$ such that $0 \leq \varphi \leq 1$ and $\varphi \equiv 1$ in a neighbourhood of $p$. For $z_{0}=\left(x_{0}, t_{0}\right)$ with some $t_{0} \in[0, \infty)$, set

$$
G_{z_{0}}(x, t)=\frac{1}{\left|t-t_{0}\right|} e^{\frac{-\left|x-x_{0}\right|^{2}}{4\left|t-t_{0}\right|}}
$$

and

$$
\Psi_{\rho}^{\alpha}\left(u, z_{0}\right)=\rho^{2 \alpha-2} \int_{T_{\rho}\left(t_{0}\right)}\left(1+|\nabla u|^{2}\right)^{\alpha} G_{z_{0}} \varphi^{2} \sqrt{|g|} d x d t
$$

where $\sqrt{|g|} d x$ is the volume element of $M$ and $T_{\rho}\left(t_{0}\right)=M \times\left[t_{0}-4 \rho^{2}, t_{0}-\right.$ $\left.\rho^{2}\right]$.

Lemma 3.2. Let $u$ be a smooth solution to (1.1) defined on $M \times[0, \infty)$ and $E_{\alpha}(u(t)) \leq E_{0}$. Then for each $z_{0} \in M \times(0,+\infty)$ and for any $0<r<$ $\rho \leq \min \left\{i_{M}, 1\right\}$, we have

$$
\Psi_{r}^{\alpha}\left(u, z_{0}\right) \leq e^{c(\rho-r)} \Psi_{\rho}^{\alpha}\left(u, z_{0}\right)+C E_{0}(\rho-r)
$$

with a uniform constant $C$ depending only on $M$ and $N$.

Proof. After a translation, we can assume $z_{0}=(0,0)$. For simplicity, we write $\Psi_{\rho}(u)=\Psi_{\rho}^{\alpha}(u, z)$ and $G=G_{(0,0)}$. Letting $u_{\rho}(y, s)=u\left(\rho y, \rho^{2} s\right)$, we obtain

$$
\Psi_{\rho}(u)=\int_{T_{1}}\left(\rho^{2}+g^{i j}(\rho y) \partial_{y_{i}} u_{\rho} \partial_{y_{j}} u_{\rho}\right)^{\alpha} G \varphi^{2}(\rho y) \sqrt{|g|}(\rho y) d y d s
$$

For simplicity, we write $g^{i j}:=g^{i j}(\rho y)$ and the same convention applies to $\varphi$, $\bar{\nabla} g^{i j}$ and so on. Here we use $\bar{\nabla}$ to denote the gradient in local coordinates; 
i.e. $\left(\frac{\partial}{\partial x_{1}}, \frac{\partial}{\partial x_{2}}\right)$, and $\tilde{\nabla}$ to denote $\left(\frac{\partial}{\partial y_{1}}, \frac{\partial}{\partial y_{2}}\right)$. Denoting $\frac{\partial}{\partial y_{i}} u_{\rho}$ by $u_{\rho, i}$, we have

$$
\begin{aligned}
\frac{d}{d \rho} \Psi_{\rho}(u)= & \int_{T_{1}} \alpha\left(\rho^{2}+g^{i j} u_{\rho, i} u_{\rho, j}\right)^{\alpha-1}\left(2 \rho+y \cdot \bar{\nabla} g^{i j} u_{\rho, i} u_{\rho, j}+2 g^{i j} u_{\rho, i} \frac{d u_{\rho, j}}{d \rho}\right) \\
& \cdot G \varphi^{2} \sqrt{|g|} d y d s+\int_{T_{1}}\left(\rho^{2}+g^{i j} u_{\rho, i} u_{\rho, j}\right)^{\alpha} G \frac{d}{d \rho}\left(\varphi^{2} \sqrt{|g|}\right) d y d s \\
\geq & -\int_{T_{1}} 2 \alpha \frac{\partial}{\partial y_{j}}\left(\sqrt{|g|}\left(\rho^{2}+g^{i j} u_{\rho, i} u_{\rho, j}\right)^{\alpha-1} g^{i j} u_{\rho, i} G\right) \frac{d u_{\rho}}{d \rho} \varphi^{2} d y d s \\
& -\int_{T_{1}} 4 \alpha\left(\rho^{2}+g^{i j} u_{\rho, i} u_{\rho, j}\right)^{\alpha-1} g^{i j} u_{\rho, i} \partial_{y_{j}} \varphi G \frac{d u_{\rho}}{d \rho} \varphi \sqrt{|g|} d y d s \\
& +\int_{T_{1}} \alpha\left(\rho^{2}+g^{i j} u_{\rho, i} u_{\rho, j}\right)^{\alpha-1} y \cdot \bar{\nabla} g^{i j} u_{\rho, i} u_{\rho, j} G \varphi^{2} \sqrt{|g|} d y d s \\
& +\int_{T_{1}}\left(\rho^{2}+g^{i j} u_{\rho, i} u_{\rho, j}\right)^{\alpha} G 2 \varphi y \cdot \bar{\nabla} \varphi \sqrt{|g|} d y d s \\
& +\int_{T_{1}}\left(\rho^{2}+g^{i j} u_{\rho, i} u_{\rho, j}\right)^{\alpha} G \varphi^{2} \frac{y \cdot \bar{\nabla}|g|}{2|g|} \sqrt{|g|} d y d s \\
= & I+I I+I I I+I V+V .
\end{aligned}
$$

By the definition of $u_{\rho}$, we have

$$
\frac{d u_{\rho}}{d \rho}=y \cdot \bar{\nabla} u+2 \rho s \frac{\partial u}{\partial t}=\frac{2 s}{\rho}\left(\frac{1}{2 s} y \cdot \tilde{\nabla} u_{\rho}+\frac{\partial u_{\rho}}{\partial s}\right)
$$

Since $u_{\rho}$ satisfies

$$
\begin{aligned}
\left(\rho^{2}+g^{i j} u_{\rho, i} u_{\rho, j}\right)^{\alpha-1} \frac{\partial u_{\rho}}{\partial s}= & \frac{\partial}{\partial y_{j}}\left(\sqrt{|g|}\left(\rho^{2}+g^{i j} u_{\rho, i} u_{\rho, j}\right)^{\alpha-1} g^{i j} u_{\rho, i}\right) \\
& +\left(\rho^{2}+g^{i j} u_{\rho, i} u_{\rho, j}\right)^{\alpha-1} A\left(u_{\rho}\right)\left(\tilde{\nabla} u_{\rho}, \tilde{\nabla} u_{\rho}\right)
\end{aligned}
$$

we have

$$
\begin{aligned}
I & \geq-2 \alpha \int_{T_{1}}\left(\rho^{2}+g^{i j} u_{\rho, i} u_{\rho, j}\right)^{\alpha-1}\left(\frac{\partial u_{\rho}}{\partial s}-\frac{g^{i j} u_{\rho, i} y_{j}}{2|s|}\right) \frac{d u_{\rho}}{d \rho} G \varphi^{2} \sqrt{|g|} d y d s \\
& \geq \int_{T_{1}} \frac{4 \alpha|s|}{\rho}\left(\rho^{2}+g^{i j} u_{\rho, i} u_{\rho, j}\right)^{\alpha-1}\left|\frac{\partial u_{\rho}}{\partial s}-\frac{y \cdot \tilde{\nabla} u_{\rho}}{2|s|}\right|^{2} G \varphi^{2} \sqrt{|g|} d y d s
\end{aligned}
$$




$$
\begin{aligned}
& -\int_{T_{1}} \frac{4 \alpha|s|}{\rho}\left(\rho^{2}+g^{i j} u_{\rho, i} u_{\rho, j}\right)^{\alpha-1}\left|\frac{g^{i j} u_{\rho, i} y_{j}}{2|s|}-\frac{y \cdot \tilde{\nabla} u_{\rho}}{2|s|}\right| \\
& \times\left|\frac{\partial u_{\rho}}{\partial s}-\frac{y \cdot \tilde{\nabla} u_{\rho}}{2|s|}\right| G \varphi^{2} \sqrt{|g|} d y d s \\
\geq & \int_{T_{1}} \frac{2 \alpha|s|}{\rho}\left(\rho^{2}+g^{i j} u_{\rho, i} u_{\rho, j}\right)^{\alpha-1}\left|\frac{\partial u_{\rho}}{\partial s}-\frac{y \cdot \tilde{\nabla} u_{\rho}}{2|s|}\right|^{2} G \varphi^{2} \sqrt{|g|} d y d s \\
& -C \int_{T_{1}} \frac{1}{\rho}\left(\rho^{2}+g^{i j} u_{\rho, i} u_{\rho, j}\right)^{\alpha-1}\left|\frac{g^{i j} u_{\rho, i} y_{j}}{2}-\frac{y \cdot \tilde{\nabla} u_{\rho}}{2}\right|^{2} G \varphi^{2} \sqrt{|g|} d y d s .
\end{aligned}
$$

Since $\left\{x_{i}\right\}$ are normal coordinates, we have $\left|g^{i j}(\rho y)-\delta^{i j}\right| \leq C \rho|y|$. The absolute value of the last term is not bigger than

$$
\begin{aligned}
& C \int_{T_{1}} \rho^{-1}\left(\rho^{2}+g^{i j} u_{\rho, i} u_{\rho, j}\right)^{\alpha-1}\left|g^{i j}(\rho y)-\delta^{i j}\right|^{2}|y|^{2}\left|u_{\rho, i}\right|^{2} G \varphi^{2} \sqrt{|g|} d y d s \\
& \quad \leq C \int_{T_{1}} \rho^{-1}\left(\rho^{2}+g^{i j} u_{\rho, i} u_{\rho, j}\right)^{\alpha-1} \rho^{2}|y|^{4}\left|u_{\rho, i}\right|^{2} G \varphi^{2} \sqrt{|g|} d y d s \\
& \quad \leq C \int_{T_{1}}\left(\rho^{2}+g^{i j} u_{\rho, i} u_{\rho, j}\right)^{\alpha}(G+C) \varphi^{2} \sqrt{|g|} d y d s \\
& \quad \leq C \Psi_{\rho}(u)+C \max _{-4 \leq s \leq-1} \int_{M} \rho^{2 \alpha-2}\left(1+|\nabla u|^{2}\right)^{\alpha} \varphi^{2} \sqrt{|g|} d x \\
& \leq C \Psi_{\rho}(u)+C E_{0},
\end{aligned}
$$

if $\rho<1$. Here we use the fact that for $s \in[-4,-1],|y|^{4} G \leq G+C$. In summary, we have

$$
\begin{aligned}
I \geq & \int_{T_{1}} \frac{2 \alpha|s|}{\rho}\left(\rho^{2}+g^{i j} u_{\rho, i} u_{\rho, j}\right)^{\alpha-1}\left|\frac{\partial u_{\rho}}{\partial s}-\frac{y \cdot \tilde{\nabla} u_{\rho}}{2|s|}\right|^{2} G \varphi^{2} \sqrt{|g|} d y d s \\
& -C \Psi_{\rho}(u)-C E_{0} .
\end{aligned}
$$

For the remaining terms, we have for a sufficiently small $\epsilon$

$$
\begin{aligned}
|I I| \leq & \epsilon \int_{T_{1}}\left(\rho^{2}+g^{i j} u_{\rho, i} u_{\rho, j}\right)^{\alpha-1} \frac{4 s^{2}}{\rho}\left|\frac{\partial u_{\rho}}{\partial s}-\frac{y \cdot \tilde{\nabla} u_{\rho}}{2|s|}\right|^{2} G \varphi^{2} \sqrt{|g|} d y d s \\
& +C(\epsilon) \int_{T_{1}} \rho^{-1}\left(\rho^{2}+g^{i j} u_{\rho, i} u_{\rho, j}\right)^{\alpha-1}\left(g^{i j} u_{\rho, i} \partial_{y_{j}} \varphi\right)^{2} \sqrt{|g|} d y d s
\end{aligned}
$$




$$
\begin{aligned}
\leq & \epsilon \int_{T_{1}}\left(\rho^{2}+g^{i j} u_{\rho, i} u_{\rho, j}\right)^{\alpha-1} \frac{4 s^{2}}{\rho}\left|\frac{\partial u_{\rho}}{\partial s}-\frac{y \cdot \tilde{\nabla} u_{\rho}}{2|s|}\right|^{2} G \varphi^{2} \sqrt{|g|} d y d s \\
& +C(\epsilon) \int_{T_{1}} \rho \sum_{k}\left|\partial_{x_{k}} \varphi\right|^{2}\left(\rho^{2}+g^{i j} u_{\rho, i} u_{\rho, j}\right)^{\alpha} \sqrt{|g|} d y d s \\
\leq & \frac{1}{2} I+C \Psi_{\rho}(u)+C E_{0}+C \max _{-4 \leq s \leq-1} \int_{M} \rho^{1+2 \alpha-2}\left(1+|\nabla u|^{2}\right)^{\alpha} \sqrt{|g|} d x \\
\leq & \frac{1}{2} I+C \Psi_{\rho}(u)+C E_{0} .
\end{aligned}
$$

Here we use again that $\rho<1$, and that $G$ is bounded on $T_{1}$. There is some constant $C$ depending only on the geometry of $M$ such that $\bar{\nabla} g^{i j}$ and $\bar{\nabla}|g|$ are bounded by $C$. Therefore, we have

$$
|I I I|+|V| \leq C \Psi_{\rho}(u)+C E_{0}
$$

and

$$
\begin{aligned}
|I V| & \leq \frac{1}{2} \Psi_{\rho}(u)+C \int_{T_{1}}\left(\rho^{2}+g^{i j} u_{\rho, i} u_{\rho, j}\right)^{\alpha}|y \cdot \bar{\nabla} \varphi|^{2} G \sqrt{|g|} d y d s \\
& \leq \frac{1}{2} \Psi_{\rho}(u)+C E_{0},
\end{aligned}
$$

where we used the fact that $|y|^{2} G$ is bounded on $T_{1}$. In conclusion, we show that for $\rho<1$,

$$
\frac{d}{d \rho} \Psi_{\rho}(u) \geq C \Psi_{\rho}(u)+C E_{0}
$$

The lemma follows from integrating this differential inequality.

With Lemmas 3.1 to 3.2 in hand, we now prove Theorem 1.2.

Proof. (i) Let $z_{i}$ be a sequence of points in $\Sigma$ which converges to $\tilde{z}=(\tilde{x}, \tilde{t})$. For any $0<R<R_{M}$, by the definition of $\Sigma$, we have

$$
\liminf _{\alpha \rightarrow 1} R^{2 \alpha-2} \int_{T_{R}\left(z_{i}\right)} \varphi^{2} e_{\alpha}\left(u_{\alpha}\right) G_{z_{i}} \sqrt{|g|} d x d t \geq \varepsilon_{0} .
$$

Since $\int_{M} e_{\alpha}\left(u_{\alpha}\right) \sqrt{|g|} d x$ is uniformly bounded by $E_{0}$, and $G_{z_{i}}$ converges to $G_{\tilde{z}}$ uniformly away from $\tilde{z}$, we can take $i \rightarrow \infty$ in the above inequality and 
switch the order of the limits to get

$$
\liminf _{\alpha \rightarrow 1} R^{2 \alpha-2} \int_{T_{R}(\tilde{z})} \varphi^{2} e_{\alpha}\left(u_{\alpha}\right) G_{\tilde{z}} \sqrt{|g|} d x d t \geq \varepsilon_{0}
$$

Since $R$ is arbitrary, we know $\tilde{z} \in \Sigma$, which shows that $\Sigma$ is closed.

The proof on the convergence of $u_{\alpha}$ outside $\Sigma$ is similar to the proof of Theorem 1.1. When $\alpha$ is fixed, we know that there is no concentration for the Dirichlet energy, so we can use the small energy condition with (2.3) to obtain a $C^{0}$-bound on the gradient as in Lemma 2.4. However, as $\alpha$ goes to 1 , the difference is that there may be a concentration point of the Dirichlet energy. But for $z \notin \Sigma$, we can find $1 / 2>R>0$ such that

$$
\liminf _{\alpha \rightarrow 1} \Psi_{R}^{\alpha}\left(u_{\alpha}, z\right)<\varepsilon_{0}
$$

We claim: There is an $\varepsilon_{0}>0$ which we use in the definition of $\Sigma$, a sequence $\left\{\alpha_{k}\right\}$, a constant $\delta>0$ and some constant $C$ depending on $N, R, E_{0}$ and $\varepsilon_{0}$ such that

$$
\left\|\nabla u_{\alpha_{k}}\right\|_{C^{0}\left(P_{\delta R}(z)\right)} \leq C
$$

Assume $z=(0,0)$ and set $r_{1}=\delta R$ for some $\delta<1 / 2$ to be determined. Suppose for all $r, \sigma \in\left(0, r_{1}\right), r+\sigma<r_{1}$ and $z_{0}=\left(x_{0}, t_{0}\right) \in P_{r}$. We will show that there are sufficiently small $R$ and $\delta$ small such that

$$
\sigma^{2 \alpha_{k}-4} \int_{P_{\sigma}\left(z_{0}\right)} e_{\alpha_{k}}\left(u_{\alpha_{k}}\right) d v d t \leq C \varepsilon_{0}
$$

for all $r>0, \sigma>0$ with $r+\sigma<r_{1}$. To see this, Lemma 3.2 implies

$$
\begin{aligned}
& \sigma^{2 \alpha_{k}-4} \int_{P_{\sigma}\left(z_{0}\right)} e_{\alpha_{k}}\left(u_{\alpha_{k}}\right) d v d t \\
& \leq C \sigma^{2 \alpha_{k}-2} \int_{P_{\sigma}\left(z_{0}\right)} e_{\alpha_{k}}\left(u_{\alpha_{k}}\right) G_{\left(x_{0}, t_{0}+2 \sigma^{2}\right)} d v d t \\
& \leq C \sigma^{2 \alpha_{k}-2} \int_{T_{\sigma}\left(t_{0}+2 \sigma^{2}\right)} \varphi^{2} e_{\alpha_{k}}\left(u_{\alpha_{k}}\right) G_{\left(x_{0}, t_{0}+2 \sigma^{2}\right)} d v d t \\
& \leq C e^{c R-\sigma} \Psi_{R}^{\alpha_{k}}\left(u_{\alpha_{k}},\left(x_{0}, t_{0}+2 \sigma^{2}\right)\right)+C E_{0}(R-\sigma) .
\end{aligned}
$$


We can choose $R$ to be small so that the last term is no larger than $\varepsilon_{0}$.

$$
\begin{aligned}
& \Psi_{R}^{\alpha_{k}}\left(u_{\alpha_{k}},\left(x_{0}, t_{0}+2 \sigma^{2}\right)\right) \\
& \quad \leq C R^{2 \alpha_{k}-2} \int_{t_{0}+2 \sigma^{2}-4 R^{2}}^{t_{0}+2 \sigma^{2}-R^{2}} \int_{M} \varphi^{2} e_{\alpha_{k}}\left(u_{\alpha_{k}}\right) G_{\left(x_{0}, t_{0}+2 \sigma^{2}\right)} d v d t \\
& \quad \leq C R^{2 \alpha_{k}-2} \int_{-4 R^{2}}^{-R^{2}} \int_{M} \varphi^{2} e_{\alpha_{k}}\left(u_{\alpha_{k}}\right) G_{\left(x_{0}, t_{0}+2 \sigma^{2}\right)} d v d t+(\delta R)^{2} E_{0} R^{-2} \\
& \quad \leq C \Psi_{R}^{\alpha_{k}}\left(u_{\alpha_{k}},\left(x_{0}, t_{0}\right)\right)+\varepsilon E_{0}+\delta E_{0} .
\end{aligned}
$$

Here in the last line we used the fact that on $T_{R}$, for any $\varepsilon>0$ we can find $\delta$ sufficiently small so that

$$
G_{\left(x_{0}, t_{0}+2 \sigma^{2}\right)} \leq C G(x, t)+\varepsilon R^{-2}
$$

(which is the exact estimate (2.18) of [4]). Hence, by choosing $\varepsilon, \delta$ properly, we have

$$
\sigma^{2 \alpha_{k}-4} \int_{P_{\sigma}\left(x_{0}, t_{0}\right)} e_{\alpha_{k}}\left(u_{\alpha_{k}}\right) d v d t \leq C \varepsilon_{0}
$$

for any $\left(x_{0}, t_{0}\right) \in P_{r}$ and any two $r, \sigma$ with $r+\sigma<r_{1}=\delta R$.

We can find $\rho \in\left[0, r_{1}\right]$ such that

$$
\left(r_{1}-\rho\right)^{2 \alpha_{k}} \sup _{P_{\rho}} e_{\alpha_{k}}\left(u_{\alpha_{k}}\right)=\max _{\sigma \in\left[0, r_{1}\right]}\left\{\left(r_{1}-\sigma\right)^{2 \alpha_{k}} \sup _{P_{\sigma}} e_{\alpha_{k}}\left(u_{\alpha_{k}}\right)\right\} .
$$

Let $\left(x_{1}, t_{1}\right)$ be the point in $P_{\rho}$ such that

$$
e_{0} \triangleq e_{\alpha_{k}}\left(u_{\alpha_{k}}\right)\left(x_{1}, t_{1}\right)=\sup _{P_{\rho}} e_{\alpha_{k}}\left(u_{\alpha_{k}}\right)
$$

If $e_{0}\left(r_{1}-\rho\right)^{2 \alpha_{k}} \leq 4$, then

$$
\left(\frac{r_{1}}{2}\right)^{2 \alpha_{k}} \sup _{P_{r_{1} / 2}} e_{\alpha_{k}}\left(u_{\alpha_{k}}\right) \leq e_{0}\left(r_{1}-\rho\right)^{2 \alpha_{k}} \leq 4
$$

which means the claim is true. Hence, we may assume that $e_{0}\left(r_{1}-\rho\right)^{2 \alpha_{k}}>4$. Set $\lambda=e_{0}^{\frac{1}{2 \alpha_{k}}}$ and

$$
v(x, t)=u_{\alpha_{k}}\left(x_{1}+\frac{x}{\lambda}, t_{1}+\frac{t}{\lambda^{2}}\right)
$$


for $(x, t) \in B_{1}(0) \times[-1,0]$. Then $v$ satisfies a scaled $\alpha$-flow equation

$$
\begin{aligned}
\left(\lambda^{-2}+|\nabla v|^{2}\right)^{\alpha_{k}-1} \partial_{t} v= & \operatorname{div}\left(\left(\lambda^{-2}+|\nabla v|^{2}\right)^{\alpha_{k}-1} \nabla v\right) \\
& +\left(\lambda^{-2}+|\nabla v|^{2}\right)^{\alpha_{k}-1} A(v)(\nabla v, \nabla v) .
\end{aligned}
$$

If we set $e(v):=\left(\lambda^{-2}+|\nabla v|^{2}\right)^{\alpha_{k}}$, then $e(v)(0,0)=1$ and

$$
\begin{aligned}
\sup _{B_{1}(0) \times[-1,0]} e(v) & \leq e_{0}^{-1} \sup _{P_{\frac{r_{1}+\left(2^{\left.1 / \alpha_{k}-1\right) \rho}\right.}{2^{1 / \alpha_{k}}}}} e_{\alpha}\left(u_{\alpha}\right) \\
& \leq e_{0}^{-1} \frac{e_{0}\left(r_{1}-\rho\right)^{2 \alpha_{k}}}{\left(r_{1}-\frac{r_{1}+\left(2^{1 / \alpha_{k}}-1\right) \rho}{2^{1 / \alpha_{k}}}\right)^{2 \alpha_{k}}} \\
& =\left(\frac{2^{1 / \alpha_{k}}}{2^{1 / \alpha_{k}}-1}\right)^{2 \alpha_{k}} \leq 5
\end{aligned}
$$

for $k$ sufficiently large. By Lemmas 3.1 and (3.5), we obtain

$$
\frac{\partial}{\partial t} e(v)-\nabla_{i}\left(a_{i j}(v) \nabla_{j} e(v)\right) \leq C e(v)
$$

where $\left(a_{i j}\right)$ is a symmetric matrix whose eigenvalues satisfy a uniform elliptic condition. By the well-known Moser iteration again, we have

$$
\begin{aligned}
1 & =e(v)(0,0) \leq C \int_{B_{1}(0) \times[-1,0]} e(v) \\
& \leq C \lambda^{4-2 \alpha_{k}} \int_{t_{1}-\lambda^{-2}}^{t_{1}} \int_{B_{1 / \lambda}\left(x_{1}\right)}\left(1+\left|d u_{\alpha_{k}}\right|\right)^{\alpha_{k}} d v d t \\
& \leq C \varepsilon_{0} .
\end{aligned}
$$

Here in the last step, we used (3.4) with $\sigma=\frac{1}{\lambda}$ and $\left(x_{0}, t_{0}\right)=\left(x_{1}, t_{0}\right)$. Since $\lambda=e_{0}^{\frac{1}{2 \alpha_{k}}}$ and $e_{0}\left(r_{1}-\rho\right)^{2 \alpha_{k}}>4$, it is easy to check that $P_{1 / \lambda}\left(x_{1}, t_{1}\right) \subset P_{r_{1}}\left(z_{0}\right)$, which is necessary for (3.4) to be true. This is a contradiction if we choose $\varepsilon_{0}$ small. This proves the claim.

We can establish higher order estimates for $u_{\alpha_{k}}$ in a smaller neighbourhood as in the proof of Theorem 1.1 so that (i) of Theorem 1.2 follows.

(ii) For any $R>0$, since $M \times\left[t^{1}, t^{2}\right]$ is compact, we can find a finite cover of $\Sigma$; i.e., $z_{i}=\left(x_{i}, t_{i}\right) \in M \times\left[t^{1}, t^{2}\right]$ such that

$$
\Sigma \cap\left(M \times\left[t^{1}, t^{2}\right]\right) \subset \bigcup_{i=1}^{l} Q_{5 R}\left(z_{i}\right)
$$

and $\left\{Q_{R}\left(z_{i}\right)\right\}$ are disjoint, where $Q_{r}(z):=B_{r}(x) \times\left(t-r^{2}, t+r^{2}\right)$. 
Let $\bar{z}_{i}=\left(x_{i}, t_{i}+R^{2}\right)$. For some constant $\delta \in(0,1 / 4)$ to be determined later, we have (by definition of $\Sigma$ and for $\alpha-1$ sufficiently small; in fact, for each $i, \alpha-1$ is required to be smaller)

$$
\begin{aligned}
& \frac{1}{2} \varepsilon_{0} \leq(\delta R)^{2 \alpha-2} \int_{t_{i}-4 \delta^{2} R^{2}}^{t_{i}-\delta^{2} R^{2}} \int_{M} \varphi^{2} e_{\alpha}\left(u_{\alpha}\right) G_{z_{i}} \sqrt{|g|} d x d t \\
& \leq C(\delta) R^{2 \alpha-4} \int_{Q_{R}\left(z_{i}\right)} e_{\alpha}\left(u_{\alpha}\right) \sqrt{|g|} d x d t \\
& +C \delta^{-2} e^{-\frac{1}{16 \delta^{2}}}(\delta R)^{2 \alpha-2} \int_{t_{i}-4 \delta^{2} R^{2}}^{t_{i}-\delta^{2} R^{2}} \int_{M} \varphi^{2} e_{\alpha}\left(u_{\alpha}\right) G_{\bar{z}_{i}} \sqrt{|g|} d x d t
\end{aligned}
$$

for $1 \leq i \leq l$. Here in the last step, we use the fact that

$$
G_{z_{i}} \leq \delta^{-2} e^{-\frac{1}{16 \delta^{2}}} G_{\bar{z}_{i}} \text { in } B_{R_{M}}\left(x_{i}\right) \times\left[t_{i}-4 \delta^{2} R^{2}, t_{i}-\delta^{2} R^{2}\right] \backslash Q_{R}\left(z_{i}\right) .
$$

By Lemma 3.2, the second term in the right-hand side of (3.5) can be estimated as

$$
\begin{aligned}
C \delta^{-2} e^{-\frac{1}{16 \delta^{2}}} \Psi_{\delta R}^{\alpha}\left(u_{\alpha}, \bar{z}_{i}\right) & \leq C \delta^{-2} e^{-\frac{1}{16 \delta^{2}}}\left(\Psi_{\sqrt{t^{1}} / 4}^{\alpha}\left(u_{\alpha}, \bar{z}_{i}\right)+E_{0}\right) \\
& \leq C\left(E_{0}, t^{1}\right) \delta^{-2} e^{-\frac{1}{16 \delta^{2}}}
\end{aligned}
$$

Hence, we can choose $\delta$ small enough so that it is smaller than $\varepsilon_{0} / 4$. Therefore,

$$
R^{2} \leq R^{4-2 \alpha} \leq C \int_{Q_{R}} e_{\alpha}\left(u_{\alpha}\right) \sqrt{|g|} d x d t
$$

Since $Q_{R}\left(z_{i}\right)$ are disjoint, we have

$$
\sum_{i=1}^{l}(5 R)^{2} \leq C \sum_{i=1}^{l} \int_{Q_{R}\left(z_{i}\right)} e_{\alpha}\left(u_{\alpha}\right) \sqrt{|g|} d x d t \leq C E_{0} .
$$

By sending $R$ to zero, we see that $\mathcal{P}^{2}\left(\Sigma \cap\left(M \times\left[t^{1}, t^{2}\right]\right)\right)$ is finite. One can prove that $\Sigma_{t}$ is a finite set in a similar way. If it is an infinite set, then we can find $l$ distinct points $x_{1}, \ldots, x_{l}$ in $\Sigma_{t}$ and $R>0$ such that balls $B_{R}\left(x_{i}\right)$ are disjoint. For the same $\delta>0$ as before, we may repeat the above argument for $\left(x_{i}, t\right)$ to see

$$
l\left(5 R^{2}\right) \leq C \sum_{i=1}^{l} \int_{Q_{R}\left(\left(x_{i}, t\right)\right)} e_{\alpha}\left(u_{\alpha}\right) \sqrt{|g|} d x d t \leq C E_{0} R^{2} .
$$

This gives an upper bound on the number of points in $\Sigma_{t}$. 
(iii) Applying the uniform bound of $\nabla u_{\alpha}$ to (1.1), the same proof of Theorem 1.1 yields that $u_{\alpha}$ converges smoothly to $u$ outside $\Sigma$. Then $u$ satisfies the harmonic map flow equation on $M \times[0, \infty) \backslash \Sigma$. The rest of the proof is exactly the same as in Theorem 7.2.3 of [17].

We now prove Theorem 1.3.

Proof. (i) Let $T$ be the first concentration time of $\Sigma$. By the local energy inequality, there is a unform constant $a>0$ such that $T \geq a>0$. In fact, there is a constant $R_{0}>0$ such that $\int_{B_{2 R_{0}}(x)} e_{\alpha}\left(u_{0}\right) d v \leq \frac{\varepsilon_{0}}{4}$ for all $x \in M$. By the local energy inequality, we have

$$
\int_{B_{R}(x)} e_{\alpha}\left(u_{\alpha}\right)(t) d v \leq \int_{B_{2 R}(x)} e_{\alpha}\left(u_{0}\right) d v+C E_{0} \frac{t}{R_{0}^{2}}<\varepsilon_{0}
$$

for $t \in[0, a]$, where the constant $a$ is independent of $\alpha$. This shows that $T \geq a>0$. Let $T_{1}$ be the first singular time of the solution $\hat{u}(x, t)$ constructed by Struwe. By uniqueness of the smooth solution of the harmonic map flow, $u(x, t)=\hat{u}(x, t)$ for $t<T \leq T_{1}$. We claim $T=T_{1}$. Otherwise, assume that $T<T_{1}$. Let $z=\left(x_{0}, T\right)$ be a most-left point of $\Sigma$; i.e. $T=\inf _{(x, t) \in \Sigma} t$. Since $T<T_{1}$, there exist $r_{1}>0$ and $r_{2}>0$ such that

$$
\Sigma \cap\left(B_{r_{1}}\left(x_{0}\right) \times\left(T-r_{1}^{2}, T\right)\right)=\emptyset
$$

and

$$
\|\nabla \hat{u}\|_{C^{0}\left(B_{r_{2}}\left(x_{0}\right) \times\left(T-r_{2}^{2}, T+r_{2}^{2}\right)\right)} \leq C_{1} .
$$

We may assume that $r_{1}>r_{2}$. There exists an $R \in\left(0, r_{2}\right)$ depending only on the constant $C$ in $(3.8)$ and $\varepsilon_{0}$ such that

$$
\left|B_{R}\left(x_{0}\right)\right|\left(1+4 C^{2}\right)^{2} \leq \varepsilon_{0} / 8
$$

Given this $R$, we then find $\rho>0$ depending only on $R, \varepsilon_{0}$ and the overall energy upper-bound $E_{0}$ such that

$$
\int_{M \backslash B_{R}\left(x_{0}\right)}\left(1+\left|\nabla u_{\alpha}\right|^{2}\right)^{\alpha}(s) e^{-\frac{\left|x-x_{0}\right|^{2}}{4(T-s)}} \varphi^{2} d v \leq \varepsilon_{0} / 8
$$

for all $s \in\left[T-4 \rho^{2}, T-\rho^{2}\right]$. Since

$$
\left(B_{R}(x) \times\left[t-4 \rho^{2}, t-\rho^{2}\right]\right) \cap \Sigma=\emptyset,
$$


by (i) of Theorem 1.2, we have for $k$ sufficiently large

$$
\left|\nabla u_{\alpha_{k}}\right|(y, s) \leq 2 C
$$

for $y \in B_{R}(x)$ and $s \in\left[t-4 \rho^{2}, t-\rho^{2}\right]$. Now we can estimate for $k$ sufficiently large

$$
\begin{aligned}
\Psi_{\rho}^{\alpha_{k}}\left(u,\left(x_{0}, T\right)\right) & =\rho^{2 \alpha_{k}-2} \int_{M \times\left[T-4 \rho^{2}, T-\rho^{2}\right]}\left(1+\left|\nabla u_{\alpha}\right|^{2}\right)^{\alpha} G_{z} \varphi^{2} d v d t \\
& \leq 3 \max _{s \in\left[T-4 \rho^{2}, T-\rho^{2}\right]} \int_{M}\left(1+\left|\nabla u_{\alpha_{k}}\right|^{2}\right)^{\alpha_{k}} e^{-\frac{|x|^{2}}{4(t-s)}} \varphi^{2} d v \\
& \leq \frac{7}{8} \varepsilon_{0},
\end{aligned}
$$

where we used (3.9), (3.10) and (3.11). This contradicts the fact that $(x, t) \in$ $\Sigma$, so this shows (i) of Theorem 1.3.

(ii) Let $\left\{\hat{p}_{i}\right\}_{i=1}^{\hat{k}}$ be the set of finite singularities of the solution $\hat{u}$ constructed by Struwe at the first singular time T. By Theorem 1.2, $u_{\alpha}$ converges smoothly to $u$ away from $\Sigma$. Set $\Sigma_{T}:=\Sigma \cap\{t=T\}=\left\{p_{i}\right\}_{i=1}^{k}$. Then, for each $x_{0} \in M \backslash \Sigma_{T}$, it follows from Theorem 1.2 that $u$ is smooth in $B_{r}\left(x_{0}\right) \times\left[T-r^{2}, T+r^{2}\right]$ for some small $r>0$. This implies that $\nabla \hat{u}$ is bounded in $B_{r}\left(x_{0}\right) \times\left[T-r^{2}, T\right)$, which means that $x_{0}$ does not belong to $\left\{\hat{p}_{i}\right\}_{i=1}^{\hat{k}}$. On the other hand, if $x_{0}$ is not a blow-up point of $\left\{\hat{p}_{i}\right\}_{i=1}^{\hat{k}}$ for $\hat{u}$ at time $T$, then there exists $r>0$ such that

$$
|\nabla u|(x, t) \leq C
$$

for $(x, t)$ in $B_{r}\left(x_{0}\right) \times\left[T-r^{2}, T\right)$. The above proof in the part (i) gives that $x_{0} \notin \Sigma_{T}$. Hence, $\left\{p_{i}\right\}$ and $\left\{\hat{p}_{i}\right\}$ are the same set of finite points; i.e., $k=\hat{k}$. Without loss of generality, assume $k=1$. It remains to show $\hat{m} \leq m$. For each $R>0$ and $\delta>0$, the local energy inequality gives

$$
\int_{B_{R}(p)} e_{\alpha}\left(u_{\alpha}\right)(T) d v \leq \int_{B_{2 R}} e_{\alpha}\left(u_{\alpha}\right)(T-\delta) d v+C E_{0} \frac{\delta}{R^{2}}
$$

For any $\varepsilon>0$, choose $R$ so that $\int_{B_{2 R}(p)} e(\hat{u})(T) \leq \varepsilon / 6$ and $\operatorname{Vol}\left(B_{2 R}(p)\right) \leq$ $\varepsilon / 6$. Let $\delta>0$ be a small number such that $C E_{0} \frac{\delta}{R^{2}} \leq \varepsilon / 6$. Taking $\alpha \rightarrow 1$ in (3.6), we have

$$
m \leq \int_{B_{2 R}(p)} e(\hat{u})(T-\delta) d v+\varepsilon / 3
$$


Finally, letting $\delta$ go to zero, we obtain

$$
m \leq \int_{B_{2 R}(p)} e(\hat{u})(T) d v+\hat{m}+\varepsilon / 3 \leq \hat{m}+\varepsilon / 2 .
$$

Theorem 1.3 follows by the arbitrariness of $\varepsilon$.

\section{An application}

In this section, we apply our results about the $\alpha$-flow to the study of minimizing sequence of the Dirichlet energy in a homotopy class. The following lemma is a variant of the main estimate in [19].

Lemma 4.1. Let $w$ be a smooth map from $B_{1}$ to $N$ satisfying the following scaled equation for some $R>0$ :

$$
\triangle w+(\alpha-1) \frac{\nabla|\nabla w|^{2} \cdot \nabla w}{R^{2}+|\nabla w|^{2}}+A(\nabla w, \nabla w)=h .
$$

Then there exists $\varepsilon_{0}>0$ such that if $E(w)<\varepsilon_{0}$ and $\alpha-1$ is sufficiently small we have

$$
\|w-\bar{w}\|_{W^{2,2}\left(B_{1 / 2}\right)} \leq C\left(\|\nabla w\|_{L^{2}\left(B_{1}\right)}+\|h\|_{L^{2}\left(B_{1}\right)}\right) .
$$

Here, $\bar{w}$ is the mean value of $w$ on $B_{1}$.

Proof. The proof is similar to the main estimate in [19]. During the proof, we write $\|\cdot\|_{p, q}$ for $\|\cdot\|_{W^{p, q}\left(B_{1}\right)}$. Multiplying the equation by $\varphi$ and taking the $L^{p}$ norm, we have

$$
\begin{aligned}
\|\triangle(\varphi w)\|_{0, p} \leq & 2(\alpha-1)\|\varphi w\|_{2, p}+C\||\nabla(\varphi w)||\nabla w|\|_{0, p} \\
& +C\|w\|_{1, p}+C\|h\|_{0, p} .
\end{aligned}
$$

For $1<p<2$, the Hölder inequality implies that

$$
\||\nabla(\varphi w)||\nabla w|\|_{0, p} \leq\|\nabla(\varphi w)\|_{0, q}\|\nabla w\|_{0,2}
$$

where $q=2 p /(2-p)$. By the $L^{p}$-estimate, for $\alpha-1$ small, we have

$$
\|\varphi w\|_{2, p} \leq C \varepsilon_{0}\|\nabla(\varphi w)\|_{0, q}+C\|w\|_{1, p}+C\|h\|_{0, p} .
$$


If we further assume that $\varepsilon_{0}$ is small, then it follows from the Sobolev embedding theorem that

$$
\|\varphi w\|_{2, p} \leq C\|w\|_{1, p}+C\|h\|_{0, p} .
$$

Setting $p=4 / 3$ and using Sobolev embedding again, we have

$$
\|\varphi w\|_{1,4} \leq C\left(\|w\|_{1,4 / 3}+\|h\|_{0,4 / 3}\right)
$$

With this, we can apply the interior $L^{2}$-estimate to (4.1) and multiply it by $\varphi$ to get

$$
\|w-\bar{w}\|_{W^{2,2}\left(B_{1 / 2}\right)} \leq C\left(\|\nabla w\|_{L^{2}\left(B_{1}\right)}+\|h\|_{L^{2}\left(B_{1}\right)}\right) .
$$

We now prove Theorem 1.4.

Proof. Since the minimizing sequence $u_{i}$ is smooth, there is a corresponding sequence $\alpha_{i}>1$ with $\alpha_{i} \rightarrow 1$ such that

$$
E_{\alpha_{i}}\left(u_{i}\right) \leq E\left(u_{i}\right)+\operatorname{Vol}(M)+\frac{1}{i}
$$

which implies

$$
\lim _{i \rightarrow \infty} E_{\alpha_{i}}\left(u_{i}\right)=\lim _{i \rightarrow \infty} E\left(u_{i}\right)+\operatorname{Vol}(M)
$$

Then there is a unique solution $u_{\alpha_{i}}(x, t)$ to the $\alpha_{i}$-flow (1.1) with initial value $u_{i}$. By Lemma 2.1, we have

$$
E_{\alpha_{i}}\left(u_{\alpha_{i}}(\cdot, 1)\right)+\int_{0}^{1} \int_{M}\left(1+\left|\nabla u_{\alpha_{i}}\right|^{2}\right)^{\alpha_{i}-1}\left|\partial_{t} u_{\alpha_{i}}\right|^{2} d v d t=E_{\alpha_{i}}\left(u_{i}\right) .
$$

Since $u_{i}$ is a minimizing sequence of $E$, we know

$$
\lim _{i \rightarrow \infty} E_{\alpha_{i}}\left(u_{\alpha_{i}}(\cdot, 1)\right) \geq \lim _{i \rightarrow \infty} E\left(u_{i}\right)+\operatorname{Vol}(M)
$$

Combining the above three identities yields

$$
\lim _{i \rightarrow \infty} \int_{0}^{1} \int_{M}\left(1+\left|\nabla u_{\alpha_{i}}\right|^{2}\right)^{\alpha_{i}-1}\left|\partial_{t} u_{\alpha_{i}}\right|^{2} d v d t=0 .
$$

Let $u$ be the weak limit of $u_{\alpha_{i}}$ in $W^{1,2}(M \times[0,1])$ by passing to a subsequence if necessary. By Theorem 1.2, we know that $u$ is a weak solution to 
the harmonic map flow. It follows from (4.4) that $u$ is a map independent of $t \in[0,1]$. Since $u_{i}(x, t)$ converges weakly to $u(x, t)$ in $W^{1,2}(M \times[0,1])$ and the trace operator $\mathbb{T}: W^{1,2}(M \times[0,1]) \rightarrow L^{2}(M \times\{0\})$ is a bounded linear operator, $u_{\alpha_{i}}(\cdot, 0)=u_{i}$ converges weakly to $u(\cdot, 0)$ in $L^{2}$. Hence, $u(\cdot, t) \equiv$ $u(\cdot, 0)$ is a (weakly) harmonic map from $M$ to $N$.

Instead of proving the energy identity for $u_{i}$ directly, we will find another minimizing sequence of maps $v_{i}$ in the same homotopy class, satisfying some perturbed harmonic map equation (see (4.7) below).

Due to (4.4), passing to a subsequence if necessary, we assume

$$
\int_{1 / 2}^{1} \int_{M}\left|\partial_{t} u_{\alpha_{i}}\right|^{2} d v d t \leq \frac{1}{16^{i}}
$$

Consider

$$
I_{i}=\left\{t \in[1 / 2,1]: \quad \int_{M}\left|\partial_{t} u_{\alpha_{i}}(\cdot, t)\right|^{2} d v \geq \frac{1}{2^{i}}\right\} .
$$

It follows from (4.5) that

$$
\left|I_{i}\right| \leq \frac{1}{8^{i}}
$$

where $\left|I_{i}\right|$ is the Lebesgue measure of $I_{i}$. Since

$$
\sum_{i=1}^{\infty}\left|I_{i}\right| \leq \sum_{i=1}^{\infty} \frac{1}{8^{i}} \leq \frac{1}{4},
$$

there exists at least one $t_{0} \in[1 / 2,1]$ such that for all $i$

$$
\int_{M}\left|\partial_{t} u_{\alpha_{i}}\left(\cdot, t_{0}\right)\right|^{2} d v \leq \frac{1}{2^{i}}
$$

For simplicity, denote $u_{\alpha_{i}}\left(x, t_{0}\right)$ by $v_{i}$ and write $h_{i}$ for $\partial_{t} u_{\alpha_{i}}\left(x, t_{0}\right)$. Hence, $v_{i}$ satisfies the equation

$$
\triangle v_{i}+2\left(\alpha_{i}-1\right)\left(\nabla^{2} v_{i}, \nabla v_{i}\right) \nabla v_{i}\left(1+\left|\nabla v_{i}\right|^{2}\right)^{-1}+A\left(\nabla v_{i}, \nabla v_{i}\right)=h_{i}
$$

As shown above, $v_{i}$ also converges weakly to $u$ in $W^{1,2}$. Moreover, by (4.3) and Lemma 2.1, $v_{i}$ is also a minimizing sequence of $E$ in the same homotypy class. Using Lemma 4.1, there exist finitely many points $x_{1}, \ldots, x_{k}$ such that $v_{i}$ converges to $u$ smoothly in $M$ away from these points. By the well-known removable singularity theorem on harmonic maps, $u$ can be extended to a smooth map on $M$. The proof of the identity (1.2) will be given in Section 5 . 
Now, let us prove the second part of Theorem 1.3 assuming that $\pi_{2}(N)$ is trivial. For simplicity, we assume $k=1$. Let $\eta(r)$ be a smooth cutoff function in $\mathbb{R}$ with the property that $\eta \equiv 1$ for $r \geq 1$ and $\eta \equiv 0$ for $r \leq 1 / 2$. For some $\rho>0$, we define a new sequence of maps $\tilde{v}_{i}: M \rightarrow N$ such that $\tilde{v}_{i}$ is the same as $v_{i}$ outside $B_{\rho}\left(x_{1}\right)$, and for $x \in B_{\rho}\left(x_{1}\right)$,

$$
\tilde{v}_{i}(x)=\exp _{u(x)}\left(\eta\left(\frac{|x|}{\rho}\right) \exp _{u(x)}^{-1} \circ v_{i}(x)\right),
$$

where exp is the exponential map on $N$. We claim that

$$
\left\|\tilde{v}_{i}-u\right\|_{W^{1,2}(M)} \rightarrow 0
$$

as $i \rightarrow \infty$. To see this, it suffices to consider $B_{\rho}\left(x_{1}\right) \backslash B_{\rho / 2}\left(x_{1}\right)$ because $\tilde{v}_{i} \equiv u$ on $B_{\rho / 2}\left(x_{1}\right)$ and $\tilde{v}_{i} \equiv v_{i}$ outside $B_{\rho}\left(x_{1}\right)$. On the other hand, Lemma 4.1 implies that $v_{i}$ converges to $u$ on $B_{\rho}\left(x_{1}\right) \backslash B_{\rho / 2}\left(x_{1}\right)$ strongly in $W^{1,2}$ and

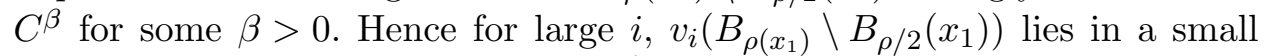
neighbourhood of $u\left(x_{1}\right)$, where $\exp _{u(x)}^{-1}$ is a well defined smooth map (if $\rho$ is small). Since $F(y)=\exp _{u(x)}\left(\eta\left(\frac{|x|}{\rho}\right) \exp _{u(x)}^{-1} y\right)$ is a smooth map from a neighbourhood of $u\left(x_{1}\right)$ into itself, we have

$$
\begin{aligned}
\left\|\tilde{v}_{i}-u\right\|_{W^{1,2}\left(B_{\rho} \backslash B_{\rho / 2}\left(x_{1}\right)\right)} & =\left\|F \circ v_{i}-F \circ u\right\|_{W^{1,2}\left(B_{\rho} \backslash B_{\rho / 2}\left(x_{1}\right)\right)} \\
& \leq C\left\|v_{i}-u\right\|_{W^{1,2}\left(B_{\rho} \backslash B_{\rho / 2}\left(x_{1}\right)\right)} \rightarrow 0 \quad \text { as } i \rightarrow \infty .
\end{aligned}
$$

Thus the claim follows. Since $\pi_{2}(N)$ is trivial, $\tilde{v}_{i}$ is in the same homotopy class as $v_{i}$. Since $v_{i}$ is a minimizing sequence of the Dirichlet energy and $v_{i}$ converges weakly to $u$ in $W^{1,2}$, we have

$$
E(u) \leq \lim _{i \rightarrow \infty} E\left(v_{i}\right) \leq \lim _{i \rightarrow \infty} E\left(\tilde{v}_{i}\right),
$$

which implies

$$
E(u)=\lim _{i \rightarrow \infty} E\left(v_{i}\right)
$$

Now, $v_{i}$ converges to $u$ strongly in $W^{1,2}(M, N)$, which means that there is no energy concentration and Lemma 4.1 in turn shows that the convergence is in $C^{\beta}$ for some $\beta>0$.

\section{An energy identity}

In the previous section, by using the $\alpha$-flow, we have constructed a sequence of $v_{i}$ satisfying 
(1) an $\varepsilon$-regularity lemma such as Lemma 4.1 holds for each $v_{i}$;

(2) $v_{i}$ minimizes $E$ within some fixed homotopy class.

The purpose of this section is to prove that for such a sequence $v_{i}$, there exist $l$ harmonic maps $\omega_{k}(k=1, \ldots, l)$ from $\mathbb{R}^{2}$ to $N$ such that

$$
\lim _{i \rightarrow \infty} E\left(v_{i}\right)=E(v)+\sum_{k=1}^{l} E\left(\omega_{k}\right)
$$

where $v$ is the weak limit of $v_{i}$ in $W^{1,2}$.

\subsection{Review of bubble tree construction}

Let us review the bubble tree construction of Parker [18]. We refer the reader to Section 1 of [18] for details.

Let $v_{i}$ be the above minimizing sequence satisfying the equation

$$
\triangle v_{i}+\frac{2\left(\alpha_{i}-1\right)\left(\nabla^{2} v_{i}, \nabla v_{i}\right) \nabla v_{i}}{1+\left|\nabla v_{i}\right|^{2}}+A\left(\nabla v_{i}, \nabla v_{i}\right)=h_{i}
$$

By the $\varepsilon$-regularity theorem, we know that $v_{i}$ converges to $v$ on any compact subset of $M \backslash\left\{x_{k}\right\}$ for finitely many bubble points $x_{k}$. A certain amount of the energy concentrates on each bubble point $x_{k}$ so that

$$
\lim _{i \rightarrow \infty}\left|\nabla v_{i}\right|^{2} d v=|\nabla v|^{2} d v+\sum_{k} m_{k} \delta_{x_{k}}
$$

At each bubble point $x_{k}$, one may find $\varepsilon_{i, k}>0$ such that $\varepsilon_{i, k} \leq \frac{1}{i}$ and

$$
\int_{B_{2 \varepsilon_{i, k}}\left(x_{k}\right)}|\nabla v|^{2} d v \leq \frac{m_{k}}{16 i^{2}}
$$

A crucial step in the Park construction [18] is using a 'renormalization constant' $C_{R}>0$, which can be chosen as small as we like. For our purposes, set $C_{R}=\varepsilon_{1} / 6$, where $\varepsilon_{1}$ is the constant in Lemma 5.4 .

By the exponential map at $x_{k}$, we may identify a point in a neighbourhood of $x_{k}$ with its geodesic coordinates. For each $k$ and $i$, the centre of 
$\operatorname{mass} c_{i, k}:=\left(c_{i, k}^{1}, c_{i, k}^{2}\right) \in M$ is defined by

$$
c_{i, k}^{j}=\frac{\int_{B_{2 \varepsilon_{i, k}}} x^{j}\left|\nabla v_{i}\right|^{2} d v}{\int_{B_{2 \varepsilon_{i, k}}}\left|\nabla v_{i}\right|^{2} d v}
$$

$\lambda_{i, k}>0$ is defined by

$$
\lambda_{i, k}=\left\{\inf \lambda: \quad \int_{B_{\varepsilon_{i, k}}\left(c_{i, k}\right) \backslash B_{\lambda}\left(c_{i, k}\right)} e\left(v_{i}\right) \leq C_{R}\right\} .
$$

This implies that

$$
\int_{B_{\varepsilon_{i, k}}\left(c_{i, k}\right) \backslash B_{\lambda_{i, k}}\left(c_{i, k}\right)} e\left(v_{i}\right)=C_{R}
$$

Set $A_{i, k}:=B_{\varepsilon_{i, k}}\left(c_{i, k}\right) \backslash B_{i \lambda_{i}, k}\left(c_{i, k}\right) \subset B_{\varepsilon_{i, k}}\left(c_{i, k}\right) \backslash B_{\lambda_{i, k}}\left(c_{i, k}\right)$. This implies that

$$
\int_{A_{i, k}} e\left(v_{i}\right) \leq C_{R}<\varepsilon_{1} / 6 \text {. }
$$

Setting $\tilde{v}_{i}(\tilde{x})=v_{i}\left(\lambda_{k, i} \tilde{x}+c_{i, k}\right)$ on $B_{i}(0)$ and passing to the limit $i \rightarrow \infty$, we have a weak limit $\omega_{k}$ (a level 1 bubble) of $\tilde{v}_{i}$ and finitely many bubble points on $\mathbb{R}^{2}$ so that the blow-up happens again at a higher level. The process stops after finite steps. Suppose there are $L$ such necks in total.

In conclusion, we have

$$
\lim _{i \rightarrow \infty} E\left(v_{i}\right)=E(v)+\sum_{k=1}^{L} E\left(\omega_{k}\right)+\limsup _{i \rightarrow \infty} \sum_{k=1}^{L} \int_{A_{i, k}} e\left(v_{i}\right) .
$$

For simplicity, we label bubbles and necks by only one index. So $A_{i, k}$ could be a neck region at any level. An example of the bubble tree and the decomposition are illustrated in figure 1. The shadow parts in figure 1(b) stand for the neck regions.

\subsection{An extension lemma}

Let $p$ be a point in $N$ and $r_{i n j}$ the injectivity radius of $N$. Consider a map $f: S^{1} \rightarrow N$ whose image lies in $B_{r_{i n j}}(p)$. Define $\tilde{f}: B_{2} \backslash B_{1} \rightarrow N$ by

$$
\tilde{f}(\theta, r)=\exp _{p}\left[(2-r) \exp _{p}^{-1} f(\theta)\right]
$$

(Here $(\theta, r)$ denotes the geodesic polar coordinates.) It is known from the definition that $\tilde{f}(\theta, 1)=f(\theta)$ and $\tilde{f}(\theta, 2)=p$. We need to compute the energy 


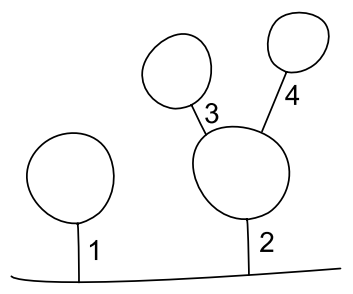

(a)

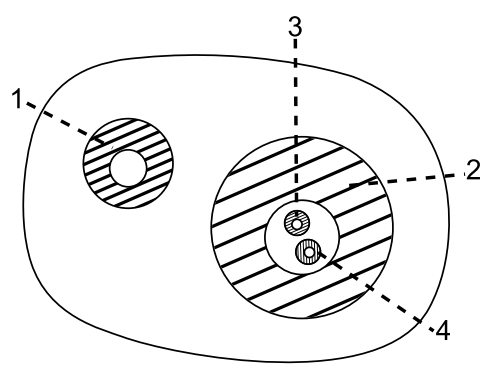

(b)

Figure 1: An example

of $\tilde{f}$. The chain rule implies

$$
\left|\frac{\partial}{\partial r} \tilde{f}(\theta, r)\right|=\left|D\left(\exp _{p}\right)_{(2-r) \exp _{p}^{-1} f(\theta)} \cdot \exp _{p}^{-1} f(\theta)\right| \leq C d(f(\theta), p)
$$

and

$$
\begin{aligned}
\left|\frac{\partial}{\partial \theta} \tilde{f}(\theta, r)\right| & =\left|D\left(\exp _{p}\right)_{(2-r) \exp _{p}^{-1} f(\theta)} \cdot(2-r) D\left(\exp _{p}^{-1}\right)_{f(\theta)} f^{\prime}(\theta)\right| \\
& \leq C(2-r)\left|f^{\prime}(\theta)\right| .
\end{aligned}
$$

Hence,

$$
E(\tilde{f}) \leq C \int_{S^{1}} \int_{1}^{2}\left(d(f(\theta), p)^{2}+\frac{1}{r^{2}}(2-r)^{2}\left|f^{\prime}(\theta)\right|^{2}\right) r d r d \theta .
$$

We summarize the computation in a lemma.

Lemma 5.1. For any $\varepsilon>0$, there exists $\eta>0$ depending only on $\varepsilon$ and $N$ such that if $f: S^{1} \rightarrow N$ satisfies

$$
\max _{\theta}\left[d(f(\theta), p)+\left|f^{\prime}(\theta)\right|\right]<\eta
$$

then the Dirichlet energy of $\tilde{f}$ defined in (5.3) on $B_{2} \backslash B_{1}$ is smaller than $\varepsilon$.

Remark 5.2. We also need a similar result for

$$
\tilde{f}(\theta, r)=\exp _{p}\left[(r-1) \exp _{p}^{-1} f(\theta)\right]
$$


which is an extension of a map defined on $\partial B_{2}$ to $B_{2} \backslash B_{1}$ such that it is a constant map on $\partial B_{1}$. The proof is the same as that of Lemma 5.1 , so we omit it.

\subsection{A reference map}

For the minimizing sequence $\left\{v_{i}\right\}$, we will construct a new minimizing sequence from $M$ to $N$ whose image looks like the weak limit map $v$ and bubbles $\omega_{k}$ connected by geodesics. It keeps a record of some topological information which will later be used to construct a new minimizing sequence.

Lemma 5.3. There exists a positive constant $\sigma$ depending only on $N$ such that if $f_{1}, f_{2}$ are two continuous maps from $\Omega$ into $N$ satisfying

$$
d\left(f_{1}(x), f_{2}(x)\right) \leq \sigma
$$

for all $x \in \Omega$ and $\left.f_{1}\right|_{\partial \Omega}=\left.f_{2}\right|_{\partial \Omega}$, then there exists a homotopy deforming $f_{1}$ to $f_{2}$ with $\partial \Omega$ fixed.

We omit the proof since it is obvious.

Lemma 5.4. There is a positive constant $\varepsilon_{1}$ depending only on $N$ such that any $W^{1,2} \cap C^{0}$ map $g: S^{2} \rightarrow N$ with $E(g)<\varepsilon_{1}$ is homotopic to a constant mapping.

This result follows immediately from [24] and is well known.

The next lemma shows how to squeeze a little room on both ends of a long neck region without changing the energy much.

Lemma 5.5. For $i$ large so that $\delta>>\lambda_{i} R$, there is a diffeomorphism $f$ from $B_{\delta} \backslash B_{\lambda_{i} R}$ to $B_{\delta / 4} \backslash B_{4 \lambda_{i} R}$ such that for any map $u: B_{\delta} \backslash B_{\lambda_{i} R} \rightarrow N$, we have

$$
1-C\left(\frac{\delta}{\lambda_{i} R}\right) \leq \frac{E\left(u \circ f^{-1}, B_{\delta / 4} \backslash B_{4 \lambda_{i} R}\right)}{E\left(u, E_{\delta} \backslash E_{\lambda_{i} R}\right)} \leq 1+C\left(\frac{\delta}{\lambda_{i} R}\right)
$$

where $C\left(\frac{\delta}{\lambda_{i} R}\right)$ is a constant which goes to zero if $\frac{\delta}{\lambda_{i} R}$ goes to infinity.

Proof. By the conformal invariance of the Dirichlet energy, $u$ can be regarded as a map from $S^{1} \times[0, K]$ to $N$, where $K=\delta /\left(\lambda_{i} R\right)$. Define $\tilde{f}: S^{1} \times[0, K] \rightarrow$ 
$S^{1} \times[4, K-4]$ by

$$
\tilde{f}(\theta, \rho)=\left(\theta, 4+\rho \frac{K-8}{K}\right) .
$$

It is straightforward to check

$$
1-C(K) \leq \frac{E\left(u \circ \tilde{f}^{-1}, S^{1} \times[4, K-4]\right)}{E\left(u, S^{1} \times[0, K]\right)} \leq 1+C(K),
$$

for some $C(K)$ such that $C(K)$ goes to zero if $K$ goes to infinity. Set $s$ : $S^{1} \times[0, K] \rightarrow B_{\delta} \backslash B_{\lambda_{i} R}$ to be

$$
s(\theta, \rho)=\left(\theta, \lambda_{i} R e^{\rho}\right) .
$$

Then $f: B_{\delta} \backslash B_{\lambda_{i} R} \rightarrow B_{\delta / 4} \backslash B_{4 \lambda_{i} R}$ defined by

$$
f=s \circ \tilde{f} \circ s^{-1}
$$

is the diffeomorphism we need.

A remark about the notation is needed for the rest of the proof. For simplicity, we omit the subscript for necks as if there was only one neck. In fact, one should repeat the construction or proof for each neck region. By the nature of the following proof, this should cause no further difficulty.

In Section 5.1, a neck region refers to $A_{i, k}=B_{\varepsilon_{i}, k}\left(c_{i, k}\right) \backslash B_{i \lambda_{i, k}}\left(c_{i, k}\right)$. For simplicity, we use a larger domain $B_{\delta} \backslash B_{\lambda_{i} R}$, omitting the centre, which is also called a neck region. By carefully choosing $\delta$ and $R$ as below, we see that the energy on a neck region is small (see (5.4) below), which is crucial to our proof. This is in fact the main reason that we use Parker's construction instead of that of Ding and Tian.

We need $\delta, R$ and $i$ to satisfy the following conditions. $\delta$ and $R$ will then be fixed for the rest of this section. We assume the neck connects the weak limit and a level 1 bubble. For a general neck, one should replace $v$ with some bubble map $\omega_{j}$.

Recall that $v_{i}$ and $v$ satisfy the identity (5.2) and let $\omega$ be one of bubbles $\omega_{k}$.

(A0) Owing to (a) and (b) in Section 5.1, choosing $\delta$ small and $R$ large, we may require

$$
\limsup _{i \rightarrow \infty} \int_{B_{\delta} \backslash B_{\lambda_{i, k} R}} e\left(v_{i}\right)<\varepsilon_{1} / 3
$$


(A1) Set $\varepsilon=\min \left\{\varepsilon_{1}, \xi / L\right\} / 8$, where $\xi$ is a positive number to be chosen in the next subsection and $L$ is the total number of necks. Lemma 5.1 gives an $\eta$. Choose $\delta$ and $R$ so that for each neck region $B_{\delta} \backslash B_{\lambda_{i} R}, v$ restricted to $\partial B_{\delta}$ satisfies

$$
\max _{\theta}|d(v, p)|+\left|\frac{d}{d \theta} v\right| \leq \min \{\eta / 2, \sigma / 2\}
$$

where $p$ is the value of $v$ at the bubble point and $\omega$ restricted to $\partial B_{R} \subset \mathbb{R}^{2}$ satisfies

$$
\max _{\theta}|d(\omega, q)|+\left|\frac{d}{d \theta} \omega\right| \leq \min \{\eta / 2, \sigma / 2\},
$$

where $q:=\lim _{y \rightarrow \infty} \omega(y)$. Fix $\delta$ and $R$ and choose some $i$ sufficiently large so that

(A2) (5.5) and (5.6) remain true if we replace $v$ by $v_{i}, \omega$ by $v_{i}\left(\lambda_{i} \cdot\right), \eta / 2$ by $\eta$ and $\sigma / 2$ by $\sigma$;

(A3) $\left|v_{i}-v\right| \leq \sigma / 3$ for $x \in M \backslash B_{\delta}$ and $\left|v_{i}\left(\lambda_{i} y\right)-\omega(y)\right| \leq \sigma / 3$ for all $y \in B_{R}$ where $\sigma$ is the constant in Lemma 5.3 ;

(A4) Let $C\left(\frac{\delta}{\lambda_{i} R}\right)$ be the function given in Lemma 5.5. For sufficiently large $i$, it satisfies $1+C\left(\frac{\delta}{\lambda_{i} R}\right)<2$.

Now we define a new map $w: M \rightarrow N$ so that

(1) $w=v_{i}$ on $M \backslash B_{\delta}$ and $B_{\lambda_{i} R}$;

(2) $w=v_{i} \circ f^{-1}$ on $B_{\delta / 4} \backslash B_{4 \lambda_{i} R}$, where $f$ is the diffeomorphism constructed in Lemma 5.5;

(3) $w(\theta, r)=\exp _{p}\left[\frac{r-\delta / 2}{\delta / 2} \exp _{p}^{-1} v_{i}(\theta, \delta)\right]$ for $(\theta, r) \in B_{\delta} \backslash B_{\delta / 2}$;

(4) $w(\theta, r)=w\left(\theta, \frac{\delta^{2}}{4 r}\right)$ for $(\theta, r) \in B_{\delta / 2} \backslash B_{\delta / 4}$;

(5) $w(\theta, r)=\exp _{q}\left[\frac{2 \lambda_{i} R-r}{\lambda_{i} R} \exp _{q}^{-1} v_{i}\left(\theta, \lambda_{i} R\right)\right]$ for $(\theta, r) \in B_{2 \lambda_{i} R} \backslash B_{\lambda_{i} R}$;

(6) $w(\theta, r)=w\left(\theta, \frac{\left(2 \lambda_{i} R\right)^{2}}{r}\right)$ for $(\theta, r) \in B_{4 \lambda_{i} R} \backslash B_{2 \lambda_{i} R}$.

It is easy to see that $w$ is homotopic to $v_{i}$. Now let us consider the map $w$ restricted to $B_{\delta / 2} \backslash B_{2 \lambda_{i} R}$. It follows from the construction that $w$ maps $\partial B_{\delta / 2}$ to $p$ and $\partial B_{2 \lambda_{i} R}$ to $q$. Hence, topologically it induces a map from $S^{2}$ to $N$ if we identify $\partial B_{2 \lambda_{i} R}$ with the north pole $P_{N}$ and $\partial B_{\delta / 2}$ with the south pole $P_{S}$. This is in fact a homotopically trivial map. To see this, consider a map $w_{1}: \mathbb{R}^{2} \rightarrow N$ which agrees with $w$ on $B_{\delta / 2} \backslash 2 \lambda_{i} R$ and maps $B_{2 \lambda_{i} R}$ to $q$ and $\mathbb{R}^{2} \backslash B_{\delta / 2}$ to $p$. By the conformal invariance of $E$, we regard $w_{1}$ as a 


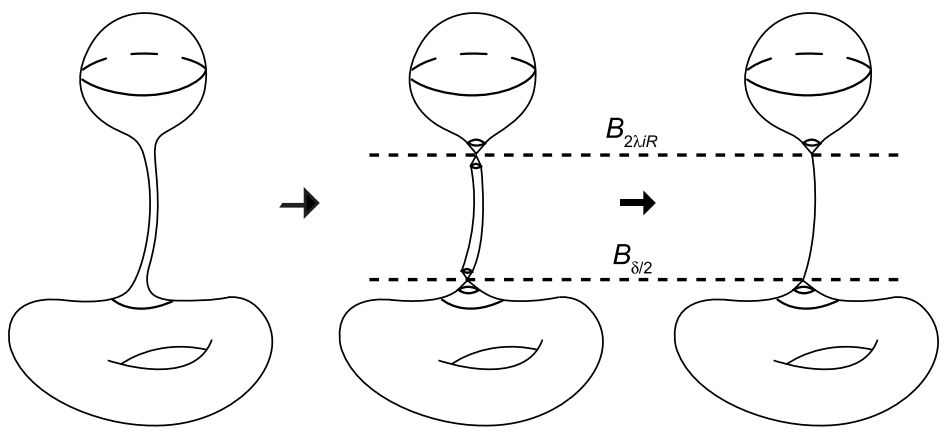

Figure 2: Reference map

map from $S^{2}$ to $N$. By (A1), (A2), (A4) and (5.4), the energy of this map is smaller than $\varepsilon_{1}$ in Lemma 5.4, hence it is homotopic to a constant map. Let $F: S^{2} \times[0,1] \rightarrow N$ be the homotopy such that $F(\cdot, 0)=w_{1}(\cdot), F(\cdot, 1) \equiv p$ and $F\left(P_{S}, t\right)=p$ for any $t \in[0,1]$. The curve $F\left(P_{N}, \cdot\right)$ connects $q$ and $p$. Let $\gamma:[0,1] \rightarrow N$ be the shortest geodesic homotopic to this curve connecting $q$ and $p$.

We can now define the reference map $\tilde{w}$. Let $\tilde{w}=w$ outside of $B_{\delta / 2} \backslash$ $B_{2 \lambda_{i} R}$, with

$$
\tilde{w}(\theta, r)=\gamma\left(\frac{\log r-\log \left(2 \lambda_{i} R\right)}{\log (\delta / 2)-\log \left(2 \lambda_{i} R\right)}\right) \quad \text { on } B_{\delta / 2} \backslash B_{2 \lambda_{i} R} \text {. }
$$

The above construction is illustrated by Figure 2. We claim that $w$ and $\tilde{w}$ are homotopic. Since $w$ and $\tilde{w}$ are the same except on $B_{2 \delta} \backslash B_{2 \lambda_{i} R}$, we construct the homotopy explicitly. For $(\theta, r) \in B_{\delta / 2} \backslash B_{2 \lambda_{i} R}$,

$$
\begin{aligned}
& G((\theta, r), s) \\
& \quad= \begin{cases}F\left(P_{N}, \frac{r-2 \lambda_{i} R}{\delta / 2-2 \lambda_{i} R}\right), & 2 \lambda_{i} R \leq r \leq r(s) \\
F\left(\left(\theta,\left(\delta / 2-2 \lambda_{i} R\right) \frac{r-r(s)}{\delta / 2-r(s)}+2 \lambda_{i} R\right), s\right), & r(s) \leq r \leq \delta / 2,\end{cases}
\end{aligned}
$$

where $r(s)=2 \lambda_{i} R+s\left(\delta / 2-2 \lambda_{i} R\right)$. From here, it is obvious that one can further deform $F\left(P_{N}, t\right)$ to $\gamma$.

If there is more than one neck region, we repeat the above construction for each neck. 


\subsection{Proof of the energy identity}

We prove the energy identity (1.2) by contradiction. Assume that (1.2) is not true. By (5.2), there exists $\xi>0$ such that

$$
\limsup _{i \rightarrow \infty} \sum_{k=1}^{L} \int_{A_{i, k}} e\left(v_{i}\right) \geq \xi
$$

Passing to a subsequence, we have

$$
\lim _{i \rightarrow \infty} \sum_{k=1}^{L} \int_{B_{\delta} \backslash B_{R \lambda_{i}}} e\left(v_{i}\right) \geq \xi .
$$

Let $\tilde{w}_{i}$ be the corresponding map of the map $v_{i}$ constructed in last subsection. We can construct a new sequence $\tilde{v}_{i}$ in the same homotopy class such that

$$
\lim _{i \rightarrow \infty} E\left(\tilde{v}_{i}\right)<\lim _{i \rightarrow \infty} E\left(v_{i}\right)
$$

More precisely, we define $\tilde{v}_{i}$ in the follows:

(1) $\tilde{v}_{i}=v_{i}$ outside the neck region $\left(B_{\delta} \backslash B_{\lambda_{i} R}\right)$;

(2) $\tilde{v}_{i}(\theta, r)=\exp _{p}\left[\frac{r-\delta / 2}{\delta / 2} \exp _{p}^{-1} v_{i}(\theta, \delta)\right]$ for $(\theta, r) \in B_{\delta} \backslash B_{\delta / 2}$;

(3) $\tilde{v}_{i}(\theta, r)=\exp _{q}\left[\frac{2 \lambda_{i} R-r}{\lambda_{i} R} \exp _{p}^{-1} v_{i}\left(\theta, \lambda_{i} R\right)\right]$ for $(\theta, r) \in B_{2 \lambda_{i} R} \backslash B_{\lambda_{i} R}$;

(4) $\tilde{v}_{i}(\theta, r)=\gamma\left(\frac{\log r-\log \left(2 \lambda_{i} R\right)}{\log (\delta / 2)-\log \left(2 \lambda_{i} R\right)}\right)$ for $(\theta, r) \in B_{\delta / 2} \backslash B_{2 \lambda_{i} R}$.

It remains to show that firstly the energy of $\tilde{v}_{i}$ in the neck region is smaller than $\xi / 2$ for $i$ large, and secondly $\tilde{v}_{i}$ is homotopic to $\tilde{w}$. For the first part, (A1) and Lemma 5.1 implies that the energy in $B_{\delta} \backslash B_{\delta / 2}$ and $B_{2 \lambda_{i} R} \backslash B_{\lambda_{i} R}$ for all necks is no more than $\xi / 4$. Moreover, the energy on the geodesic part is

$$
E\left(\tilde{v}_{i} ; B_{\delta / 2} \backslash B_{2 \lambda_{i} R}\right)=\frac{\operatorname{Length}(\gamma)^{2}}{\log (\delta / 2)-\log \left(2 \lambda_{i} R\right)},
$$

which can be made as small as we like when $i$ is sufficiently large due to the fact that the length of $\gamma$ is fixed (see Section 5.3).

For the second point, if there is only one neck, then $M$ is decomposed into three parts; $M \backslash B_{\delta / 2}, B_{\delta / 2} \backslash B_{2 \lambda_{i} R}$ and $B_{2 \lambda_{i} R}$. For the reference map $\tilde{w}$, the decomposition is fixed because we chose and fixed a large $i$ in the construction. For $\tilde{v}_{i}$, the centre of $B_{\delta}$ and $B_{\lambda_{i} R}$ changes with $i$ and so does 
the radius of $B_{2 \lambda_{i} R}$. However, when restricted to the separating circles $\partial B_{\delta / 2}$ and $\partial B_{2 \lambda_{i} R}, \tilde{w}$ and $\tilde{v}_{i}$ are constant maps to the same points. This allows us to prove the homotopy between $\tilde{w}$ and $\tilde{v}_{i}$ on each of the three parts separately.

On $M \backslash B_{\delta / 2}$, (A1) and (A3) show that $d\left(\tilde{w}, \tilde{v}_{i}\right)<\sigma$, which implies that there is a homotopy between them leaving $\partial B_{\delta / 2}$ fixed. The bubble part $B_{2 \lambda_{i} R}$ of $\tilde{w}_{i}$ and $\tilde{v}_{i}$ are homotopic for the same reason. On the $B_{\delta / 2} \backslash B_{2 \lambda_{i} R}$ part, they are different parametrizations of the same curve. This concludes our proof for (1.2) in the case of only one neck.

For the general case of multiple necks, the decomposition is more complicated but the same argument applies. To illustrate the idea, consider a bubble tree as shown in Figure 1(a). The necks are labelled from 1 to 4 . The corresponding decomposition of $M$ is illustrated in Figure 1(b). The shadow parts are neck regions. There are eight circles separating the neck regions and the bubble regions. Both $\tilde{w}_{i}$ and $\tilde{v}_{i}$ map these circles to $p_{i}$ 's and $q_{i}$ 's. The homotopy for bubble regions (including $M \backslash\left(B_{\delta / 2}\left(x_{1}\right) \cup B_{\delta / 2}\left(x_{2}\right)\right)$ follows from Lemma 5.3 and the homotopy for neck regions is obvious because they are different parametrization of the same geodesics.

\section{A. Appendix}

In this appendix, we prove the local existence and uniqueness of the $\alpha$-flow equation

$$
\partial_{t} u=\triangle_{M} u+(\alpha-1) \frac{\nabla|\nabla u|^{2} \cdot \nabla u}{1+|\nabla u|^{2}}+A(u)(\nabla u, \nabla u),
$$

for any smooth initial value $u_{0}: M \rightarrow N \subset \mathbb{R}^{k}$.

As in the case of harmonic maps, there is an intrinsic way of writing the right-hand side of (A.1). If we regard $\left(1+|\nabla u|^{2}\right)^{\alpha-1} \nabla u$ as a section of the pull-back bundle $u^{*} T N$ and denote the induced connection of $u^{*} T N$ by $\tilde{D}$, the right-hand side can be written as

$$
\tau_{\alpha}(u):=\frac{1}{\left(1+|\nabla u|^{2}\right)^{\alpha-1}} \sum_{i} \tilde{D}_{e_{i}}\left(\left(1+|\nabla u|^{2}\right)^{\alpha-1} \nabla_{e_{i}} u\right),
$$

where $\left\{e_{i}\right\}$ is an orthonormal basis of $T M$ in some open set. In the following, we will call $\tau_{\alpha}$ the $\alpha$-tension field of $u$. It is obvious that smooth solution to (A.1) is equivalent to that of

$$
\frac{\partial u}{\partial t}=\tau_{\alpha}(u),
$$


where we regard $u(\cdot, t)$ as maps into $N$. This allows us to consider a totally geodesic isometric embedding of $N$ into $\mathbb{R}^{k}$ with some non-flat metric as in [10]. Precisely, there is a metric $h$ on $\mathbb{R}^{k}$ and an embedding $N$ into $\mathbb{R}^{k}$, such that the isometric embedding is totally geodesic, and there exists an isometric involution $i$ which acts on a tubular neighbourhood $\mathcal{T}$ of $N$, leaving points of $N$ fixed. By composing with this embedding, we regard $u$ as a map from $M$ into $\left(\mathbb{R}^{k}, h\right)$, and denote by $\tau_{\alpha}^{N}$ and $\tau_{\alpha}^{\mathbb{R}^{k}}$ the $\alpha$-tension fields of $u$ as a map into $N$ and into $\mathbb{R}^{k}$ respectively. Since $N$ is a totally geodesic submanifold, we have $\tau_{\alpha}^{N}(u)=\tau_{\alpha}^{\mathbb{R}^{k}}(u)$ (see page 108 in [10]). Therefore, it suffices to study

$$
\frac{\partial u^{\beta}}{\partial t}=\triangle_{M} u^{\beta}+(\alpha-1) \frac{\nabla|\nabla u|^{2} \cdot \nabla u^{\beta}}{1+|\nabla u|^{2}}+\Gamma(u) \# \nabla u \# \nabla u,
$$

where $u^{\beta}$ are the components of $u, \triangle_{M}$ is the usual Laplacian on $M$ and $\Gamma$ is the Christoffel symbol of the Levi-Civita connection of $\left(\mathbb{R}^{k}, h\right)$. Since $|\nabla u|^{2}=h_{\beta \gamma}(u) \nabla u^{\beta} \cdot \nabla u^{\gamma}$, when we expand $\nabla|\nabla u|^{2}$, there is an extra term involving the gradient of $h(u)$ which can be absorbed into the last term. The equation can be rewritten as

$$
\frac{\partial u^{\beta}}{\partial t}=\triangle_{M} u^{\beta}+2(\alpha-1) \frac{\nabla_{i j}^{2} u^{\gamma} \nabla_{i} u^{\gamma} \nabla_{j} u^{\beta}}{1+|\nabla u|^{2}}+\tilde{\Gamma}(u) \# \nabla u \# \nabla u
$$

Next, we start a routine iteration procedure to show the local existence of (A.3). Consider the following linear parabolic system:

$$
\frac{\partial u}{\partial t}=\Delta u+(\alpha-1) \frac{\left(\nabla^{2} u, \nabla v\right) \nabla v}{1+|\nabla v|^{2}}+\tilde{\Gamma}(v) \# \nabla v \# \nabla v
$$

Let $w=u-u_{0}$. Then $w$ satisfies

$$
\begin{aligned}
\frac{\partial w}{\partial t}= & \Delta w+(\alpha-1) \frac{\left(\nabla^{2} w, \nabla v\right) \nabla v}{1+|\nabla v|^{2}}+\tilde{\Gamma}(v) \# \nabla v \# \nabla v \\
& +\triangle u_{0}+(\alpha-1) \frac{\left(\nabla^{2} u_{0}, \nabla v\right) \nabla v}{1+|\nabla v|^{2}}
\end{aligned}
$$

For some $\mu \in(1,2)$, set

$$
V=\left\{v \in C^{\mu, \mu / 2}(M \times[0, T]) \mid \quad\|v\|_{C^{\mu, \mu / 2}(M \times[0, T])} \leq 1, v(0) \equiv 0\right\} .
$$


For each $v \in V$, the Schauder estimate of linear parabolic systems (see [8] and [20]) implies

$$
\|w\|_{C^{\mu+1, \frac{\mu+1}{2}}(M \times[0, T])} \leq C\left(\|w\|_{C^{0}(M \times[0, T])}+\left\|u_{0}\right\|_{C^{\mu+1}(M)}+1\right) .
$$

Since $w(\cdot, 0) \equiv 0$, we have

$$
\|w\|_{C^{0}(M \times[0, T])} \leq T C\left(\|w\|_{C^{0}(M \times[0, T])}+\left\|u_{0}\right\|_{C^{\mu+1}(M)}+1\right) .
$$

Choose $T$ small so that

$$
\|w\|_{C^{0}(M \times[0, T])} \leq T C\left(\left\|u_{0}\right\|_{C^{\mu+1}(M)}+1\right) .
$$

By the interpolation of the Hölder space, for $\sigma>0$ such that $\mu=(1-\sigma)(\mu+$ 1), we have

$$
\|w\|_{C^{\mu, \mu / 2}(M \times[0, T])} \leq\|w\|_{C^{0}(M \times[0, T])}^{\sigma}\|w\|_{C^{\mu+1, \frac{\mu+1}{2}}(M \times[0, T])}^{1-\sigma} .
$$

This implies that we can choose $T$ sufficiently small so that $w \in V$.

Now start from any $v_{0} \in V$. Let $v_{k+1}$ be the solution of (A.4) with $v=v_{k}$ and zero initial value. We then have a uniform bound

$$
\left\|v_{k}\right\|_{C^{\mu+1, \frac{\mu+1}{2}}(M \times[0, T])} \leq C .
$$

Using Schauder estimate again, we obtain a uniform $C^{\mu+3, \frac{\mu+3}{2}}$ estimate for $v_{k}$. By taking a subsequence, we know $v_{k}$ converges to some $w$ in $C^{\mu+1, \frac{\mu+1}{2}}$ $(M \times[0, T])$. Then $w+u_{0}$ is a solution to the $\alpha$-flow with initial value $u_{0}$.

If there are two smooth solutions $u$ and $w$ to Equation (A.3) defined on $[0, T]$. We can subtract the two equations, multiply both sides by $u-w$ and integrate over $M$ to get

$$
\begin{aligned}
\frac{1}{2} \frac{d}{d t} \int_{M}|w-u|^{2} d v \leq & -\int_{M}|\nabla(u-w)|^{2} d v \\
& +2(\alpha-1) \int_{M}\left(\nabla_{i j}^{2}\left(u^{\beta}-w^{\beta}\right)\right) \frac{u_{i}^{\beta} u_{j}^{\gamma}}{1+|\nabla u|^{2}}\left(u^{\gamma}-w^{\gamma}\right) d v \\
& +C \int_{M}|u-w|^{2} d v+C \int_{M}|\nabla u-\nabla w||u-w| d v
\end{aligned}
$$

For the second term on the right-hand side, integrating by parts and noticing that

$$
\frac{u_{i}^{\beta} u_{j}^{\gamma}}{1+|\nabla u|^{2}} \nabla_{i}\left(u^{\beta}-w^{\beta}\right) \nabla_{j}\left(u^{\gamma}-w^{\gamma}\right) \geq 0
$$


yields

$$
\frac{d}{d t} \int_{M}|u-w|^{2} d v \leq C \int_{M}|u-w|^{2} d v
$$

which implies $u \equiv w$ on $M \times[0, T]$ if $u=w$ for $t=0$. It follows immediately from the uniqueness and the involution isometry $i: \mathcal{T} \rightarrow \mathcal{T}$ that if $u_{0}$ is a map from $M$ to $N$, the image of the local solution $u(x, t)$ obtained above lies in $N$.

\section{Acknowledgments}

After finishing an early version in 2010, the authors have received useful comments and suggestions from Professors R. Schoen, R. Hamilton and M. Struwe and would like to thank them for their interests and encouragements. The research of the first author was supported by the Australian Research Council grant no. DP0985624. The second author was supported by a postdoctoral fellowship at the University of Queensland through the grant no. DP0985624. The second author was also supported by NSFC No. 11101272.

\section{References}

[1] M. Bertsch, R. Dal Passo and R. Van der Hout, Nonuniqueness for the heat flow of harmonic maps on the disk, Arch. Ration. Mech. Anal., 161(2) (2002), 93-112.

[2] K.C. Chang, W.Y. Ding and R. Ye, Finite-time blow-up of the heat flow of harmonic maps from surfaces, J. Differ. Geom. 36(2) (1992), $507-515$.

[3] J.Y. Chen and G. Tian, Compactification of moduli space of harmonic mappings, Comment. Math. Helv. 74 (1999), 201-237.

[4] Y.M. Chen and M. Struwe, Existence and partial regularity for heat flow for hamonic maps, Math. Z. 201 (1989), 83-103.

[5] W.Y. Ding and G. Tian, Energy identity for a class of approximate harmonic maps from surfaces, Commun. Anal. Geom. 3 (1996), 543-554.

[6] F. Duzaar and E. Kuwert, Minimization of conformal invariant energies in homotopy classes, Calc. Var. Partial Differ. Eq. 6(4) (1998), 285-313.

[7] J. Eells and J.H. Sampson, harmonic mappings of Riemannian manifolds, Amer. J. Math. 86 (1964) 109-160. 
[8] S.D. Eidel'man, Parabolic systems, Translated from the Russian by Scripta Technica, London North-Holland Publishing Co., AmsterdamLondon; Wolters-Noordhoff Publishing, Groningen 1969.

[9] M. Giaquinta, M.-C. Hong and H. Yin, A New Approximation of Relaxed Energies for Harmonic Maps and the Faddeev Model, Calc. Var. Partial Differ. Eq. 41 (2011) 45-69.

[10] R.S. Hamilton, Harmonic maps of manifolds with boundary, Lecture Notes in Mathematics, 471, Springer-Verlag, Berlin-New York, 1975.

[11] T. Lamm, Energy identity for approximations of harmonic maps from surfaces, Trans. Amer. Math. Soc. 362 (2010), 4077-4097.

[12] L. Lemaire, Applications harmoniques des surfaces Riemanniennes, J. Differ. Geom. 13 (1978), 51-78.

[13] J.Y. Li and G. Tian, The blow-up locus of heat flows for harmonic maps, Acta Math. Sin. (Engl. Ser.), 16(1) (2000), 29-62.

[14] Y.X. Li and Y.D. Wang, A weak energy identity and the length of necks for a sequence of Sacks-Uhlenbeck $\alpha$-harmonic maps, Adv. Math. 225(3) (2010), 1134-1184, doi:10.1016/j.aim.2010.03.020.

[15] Y.X. Li and Y.D. Wang, Bubbling location for sequences of approximate f-harmonic maps from surfaces; Internat. J. Math. 21(4) (2010), 475-495.

[16] F.H. Lin and C.Y. Wang, Harmonic and quasi-harmonic spheres, Comm. Anal. Geom. 7(2) (1999), 397-429.

[17] F.H. Lin and C.Y. Wang, The analysis of harmonic maps and their heat flows, World Scientific Publishing Co. Pte. Ltd. (2008).

[18] T. Parker, Bubble tree convergence for harmonic maps, J. Differ. Geom. 44 (1996), 595-633.

[19] J. Sacks and K. Uhlenbeck, The existence of minimal immersions of 2-spheres, Ann. Math. (2), 113(1) (1981), 1-24.

[20] W. Schlag, Schauder and $L^{p}$ estimates for parabolic systems via companato spaces, Commun. Partial Differ. Eq. 21(7-8) (1996), 1141-1175.

[21] R. Schoen, Analytic aspects of the harmonic map problem, Seminar on Nonlinear Partial Differential Equations (Berkeley, California, 1983), 321-358, Math. Sci. Res. Inst. Publ., 2, Springer, New York, 1984. 
[22] R. Schoen and S.T. Yau, Existence of incompressible minimal surfaces and the topology of three dimensional manifolds with non-negative scalar curvature, Ann. Math. 110 (1979), 127-142.

[23] M. Struwe, On a free boundary problem for minimal surfaces, Invent. Math. 75 (1984), 547560.

[24] M. Struwe, On the evolution of harmonic maps of Riemannian surfaces, Comm. Math. Helv. 60 (1985), 558-581.

[25] M. Struwe, On the evolution of harmonic maps in higher dimensions, J. Differ. Geom. 28 (1988), 485-502.

[26] P. Topping, Reverse bubbling and nonuniqueness in the harmonic map flow, Int. Math. Res. Not. (10) (2002), 505-520.

[27] K. Uhlenbeck, Regularity for a class of non-linear elliptic systems, Acta Math. 138 (1977), 219-240.

[28] K. Uhlenbeck, Morse theory by perturbation methods with applications to harmonic maps, Trans. Amer. Math. Soc 267 (1981), 569-583.

Department of Mathematics,

The University of QueEnsland

BRISBANE, QLD 4072,

Australia

E-mail address: hong@maths.uq.edu.au

School of Mathematical Sciences, University of Science And Technology of China, Hefei, China

E-mail address: haoyin@ustc.edu.cn

ReCEIVED July 7, 2011 
